\title{
WestVirginiaUniversity
}

THE RESEARCH REPOSITORY @ WVU

Graduate Theses, Dissertations, and Problem Reports

2007

\section{Comparative study in stereographic reading}

Alberto E. Santiago

West Virginia University

Follow this and additional works at: https://researchrepository.wvu.edu/etd

\section{Recommended Citation}

Santiago, Alberto E., "Comparative study in stereographic reading" (2007). Graduate Theses,

Dissertations, and Problem Reports. 1864.

https://researchrepository.wvu.edu/etd/1864

This Thesis is protected by copyright and/or related rights. It has been brought to you by the The Research Repository @ WVU with permission from the rights-holder(s). You are free to use this Thesis in any way that is permitted by the copyright and related rights legislation that applies to your use. For other uses you must obtain permission from the rights-holder(s) directly, unless additional rights are indicated by a Creative Commons license in the record and/ or on the work itself. This Thesis has been accepted for inclusion in WVU Graduate Theses, Dissertations, and Problem Reports collection by an authorized administrator of The Research Repository @ WVU. For more information, please contact researchrepository@mail.wvu.edu. 


\title{
Comparative Study in Stereographic Reading
}

\author{
Alberto E. Santiago \\ Thesis submitted to the \\ College of Engineering and Mineral Resources \\ at West Virginia University \\ in partial fulfillment of the requirements \\ for the degree of \\ Master of Science \\ in \\ Computer Science
}

Dr. Frances L. Van Scoy, Chair Dr. Yenumula V. Reddy Dr. Raymond Morehead Lane Department of Computer Science and Electrical Engineering

\author{
Morgantown, West Virginia
}

2007

Keywords: Stereographic Reading, Memory, Autostereogram generation (c)2007, Alberto E. Santiago 


\begin{abstract}
Comparative Study in Stereographic Reading

Alberto E. Santiago

Viewing a Single Image Random Dot Stereogram (SIRDS) or autostereogram picture requires careful concentration from the viewer. The concentration necessary to perceive depth may affect the performance of the reading process. We are studying if information perceived by way of a stereogram is better retained in memory compared to normal reading without stereo vision. To measure subject's memory performance we administered a questionnaire after a stereographic experience containing a readable story. We discovered that exposing subjects to stereographic information has a slight advantage on the ability to remember precise details of the information read.
\end{abstract}




\section{Contents}

1 Introduction 1

1.1 History of the Autostereograms . . . . . . . . . . . . . . . . . 1

1.2 Alternative stereographic tools . . . . . . . . . . . . . . . . . . 3

2 Depth Perception Cues 5

3 Motivation $\quad 7$

4 Learning to see Random Dot Stereograms $\quad 8$

4.1 Instructions to see the stereogram . . . . . . . . . . . . . . . . . 9 9

4.2 Limitations of the SIRDS . . . . . . . . . . . . . . . . . 10

5 Creating the Autostereogram $\quad 12$

5.1 Calculation for Projection Pixels . . . . . . . . . . . . . . 12

5.2 Pixel color constraints . . . . . . . . . . . . . . . . . . . . . . 14

5.3 Program implementation issues . . . . . . . . . . . . . . . . 14

5.3.1 Flat layers in the autostereogram (depth steps) . . . . . . . . 15

5.3 .2 Avoiding 3-D artifacts . . . . . . . . . . . . . . . . 19

5.3 .3 Custom font set . . . . . . . . . . . . . . . . 19

5.4 Software functionality . . . . . . . . . . . . . . . 22

6 Previous Work $\quad 22$

7 Method 22

7.1 Target story and questionnaire . . . . . . . . . . . . . . . 23

7.1.1 Nature of the questions . . . . . . . . . . . . . . . . . 23

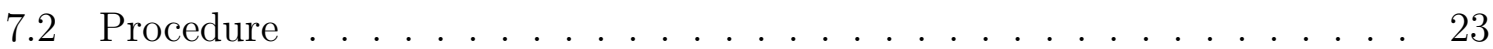

7.3 how long does it take to see the illusion? . . . . . . . . . . . . 24

8 Results $\quad 24$

9 Discussion $\quad 27$

9.1 Notes during the experiments . . . . . . . . . . . . . . . . . 28

9.2 Memory recall versus level of comfort . . . . . . . . . . . . . . . . 28

10 Final Remarks $\quad 29$

10.1 Venues for future research . . . . . . . . . . . . . . . . . 30

A Stereo disparity as a tool to ascertain similarities or differences 33

B Code Listing 


\section{List of Figures}

1 Stereogram of a box created with two monocular pictures . . . . . . . . 2

2 Wheatstone's stereoscope . . . . . . . . . . . . . . 2

3 Examples of autostereograms . . . . . . . . . . . . . . . . 4

4 red-cyan anaglyph of Mars Rover . . . . . . . . . . . . . . 5

$5 \quad$ Illustrating stereo disparity . . . . . . . . . . . . . . . . . . 8

6 Helper dots (stereo visual aids) . . . . . . . . . . . . . . . . 10

$7 \quad$ SIRDS horizontal disparity constraint defeated by rotation . . . . . . . 11

8 Vantage points on a pyramid that point to the viewer . . . . . . . . . . 12

9 Geometrical projection model . . . . . . . . . . . . . . . 13

10 Shared projection points on image plane . . . . . . . . . . . . . 15

11 Improving 3-D surface homogeneity . . . . . . . . . . . . . . . . . . 18

12 Avoiding artifacts from forming in 3-D surface . . . . . . . . . . . . 20

13 Adjusting the $3-\mathrm{D}$ fonts . . . . . . . . . . . . . . . . . . 21

14 Font alphabet . . . . . . . . . . . . . . . . . . . 21

15 Noticing differences among similarities . . . . . . . . . . . . . . 33

16 Consolidating similarities among dissimilar objects . . . . . . . . . . 34

17 Using a text stereogram to emphasize words . . . . . . . . . . . . . . 34

\section{List of Tables}

1 Visual depth cues . . . . . . . . . . . . . . . . . . 6

2 Mapping pixel depth to projection separation . . . . . . . . . 16

3 Questionnaire answer tabulation . . . . . . . . . . . . . . . 25

4 Group score statistics . . . . . . . . . . . . . . . 26

5 Comfort level distribution for stereographic readers . . . . . . . . . . . 26 


\section{Introduction}

Single image random dot stereograms (SIRDS) or "autostereograms" are more than computer generated expressions of art. SIRDS have been used by researchers in many fields of science including Psychology, Neurology and Visualization. From measuring neurological responses to serving as a visualization tool for interactive applications, the stereogram is vastly utilized. In this paper we examine another potential application for the stereogram by testing whether or not its use has any influence on human memory.

To put the topic in perspective, we dedicate section 1 to a brief historical background on the stereogram and additional tools used for three dimensional representations; while section 2 discusses depth perception cues. Sections 4 and 5 introduce the motivation for this research and provide detailed descriptions on stereopsis and how it is achieved. Section 6 discusses the implementation issues and processes involved with the creation of autostereograms in a computer. In sections 7-8 we describe the experiment performed for this study and results gathered from the experiences. Section 9 provides a discussion on our findings and we conclude the paper with suggested venues for future research.

\subsection{History of the Autostereograms}

The single image random dot stereogram was preceded historically by the stereogram. It is important to note that the words stereogram and autostereogram denote two distinct 3-D perception tools, even though culturally the terms are incorrectly interchanged. The stereogram consists of a pair of monocular images ${ }^{1}$ that represent a particular object looked from the two distinct perspectives of the left and right eyes. These two images are meant to be looked at dichoptically, i.e. the left eye gazes at the left picture at the same time that the right eye gazes at the right picture. Figure 1 shows an example of a stereogram where two flat pictures create the illusion of a 3-D box. When both images are looked at dichoptically, the Human Visual System (HVS) is able to merge both images into a congruent three dimensional object, and relief can be perceived.

The earliest account of stereograms was in 1838 when the British scientist and inventor Charles Wheatstone observed and described the phenomenon of binocular vision. In his publication on Contributions to the Physiology of Vision [Wheatstone 1838], he argued that the perspective of objects held immediately before an spectator looked different from the left and right eye vantage points. At this point he realized that images of the same object, even though dissimilar from the angle of the optic axes, could be fused in the mind as a solid object. From this, he decided to replicate this phenomenon by drawing two images that represented left and right eye perspectives of an object. Using a stereoscope, a devise he invented to aid the eyes into perceiving the two images simultaneously, he could produce an illusion of a solid object from the two flat images. Figure

\footnotetext{
${ }^{1}$ the term monocular image refers to an image that can be seen with one eye and has no stereo effect, e.g. picture, drawing, painting, etcetera
} 


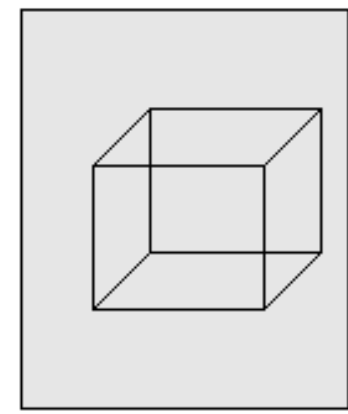

Left Eye Image

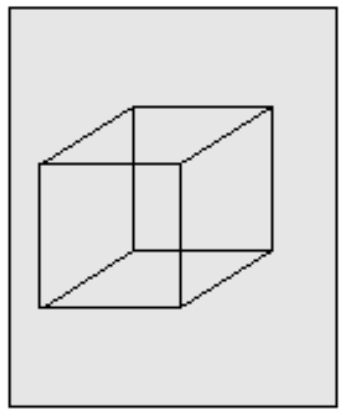

Right Eye Image

Figure 1: Stereogram of a box created with two monocular pictures

2 shows Wheatstone's stereoscope (reproduced from fig. 8 on [Wheatstone 1838]). The stereoscope allowed the viewer to fuse both images effortlessly without having to diverge eyes but by looking at the reflection of the images on the mirror. In terms of functionality, today's haploscopes are homologous to the early stereoscope.

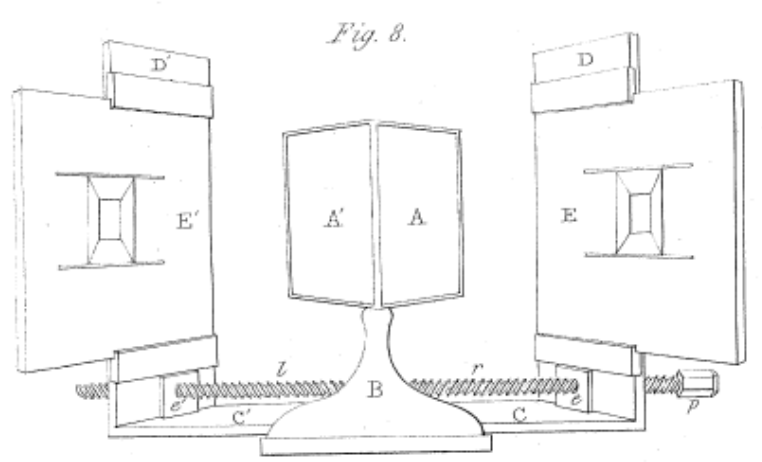

Planes A and A' represent mirrors that reflect images held in place by boards E and E'. These vertical boards can slide along planes D and D' to adjust the reflection position. Screw 'p' regulates the separation distance between the images and the mirrors; both image panels move simultaneously as 'p' is adjusted. $\mathrm{C}$ and $\mathrm{C}$ ' are disjoint pieces that join or separate image panels corresponding to the rotation of 'p'.

Figure 2: Wheatstone's stereoscope

The advent of the random dot stereogram (RDS) substituted the two monocular images with patterns of random dots. Bela Julesz is attributed with inventing and popularizing the use of computer-generated random dot stereograms. In 1959, Julesz himself 
claimed to have created "[stereograms] monocularly devoid of all shapes and contours" [Julesz 1972]. Although similar stereograms of random patterns had been used in the past ${ }^{2,3}$ it was Julesz who popularized their use, particularly in the field of psychophysiology and neurophysiology.

Random dot stereograms evolved into SIRDS in 1979 when Christopher Tyler discovered that the same stereo effect could be obtained by repeating patterns of random dots on a single image. Subsequently, SIRDS dispensed with the random dots and evolved into colorful images of repeating monocular patterns that also conveyed the stereo effect. SIRDS became Single Image Stereograms (SIS), hollusions, or Autostereograms as we popularly call them nowadays.

Figure 3 illustrates the two types of stereograms we can created using our software. See section 5.4 for a detailed discussion on software functionality created for this study.

\subsection{Alternative stereographic tools}

Apart from the stereograms, a variety of tools exist that use binocular parallax to induce three dimensional perspective. Unlike the stereograms, the main disadvantages of these alternatives is that they are expensive and involve the use of special equipment to perceive stereo imagery. Time multiplexed methods for producing the stereo effect require the alternation between the left and right eye images. Examples of time multiplexed method are the Cave and ImmersaDesk ${ }^{4}$ immersive environments, in which LCD shutter glasses synchronize the images projected to both eyes.

The stereo effect can also be produced using the time parallel method in which both images are displayed at once. Time parallel techniques are useful when there are two independent screens (as the case with head mounted displays), but the images can also be splitted using color filters or polarized lenses. Anaglyphs are examples of this stereo technique. An anaglyph consists of a pair of superimposed monocular images that correspond to left and right eye vantage points of a scene. The left image is displayed only with the red intensity component, while the right image is displayed with the cyan intensity component. Both images are then merged together with a slight offset. Looking through the anaglyphoscope (normally a pair of inexpensive glasses with redgreen filters) the left eye's red filter allows the eye to see only the cyan component, while the cyan filter allows the left eye to see only the red component. The filtered images

\footnotetext{
${ }^{2}$ In 1901 Santiago Ramon y Cajal described a technique for hiding stereographic messages on a background of random dots.[Guest editorial, Perception 2006 volume 35 pages 1-8]

${ }^{3}$ In 1939, Boris Kompaneyski created two oval figures filled with random dots that when fused binocularly produced the image of a face. Claus Aschenbrenner was also able to hide text using random dot stereograms in 1954.[González 2000]

${ }^{4}$ The Electronic Visualization Laboratory at the University of Illinois at Chicago invented both of these virtual reality tools in 1992 and 1995 respectively. http://www.evl.uic.edu
} 


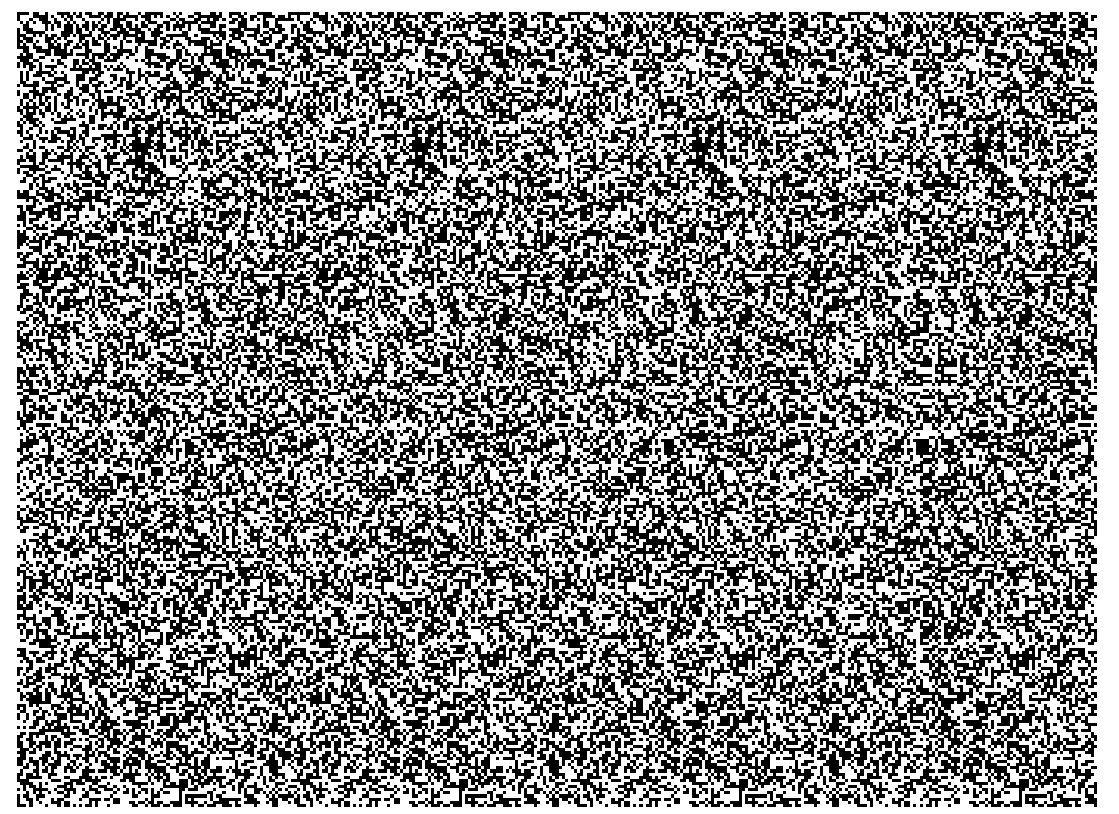

(a) SIRDS

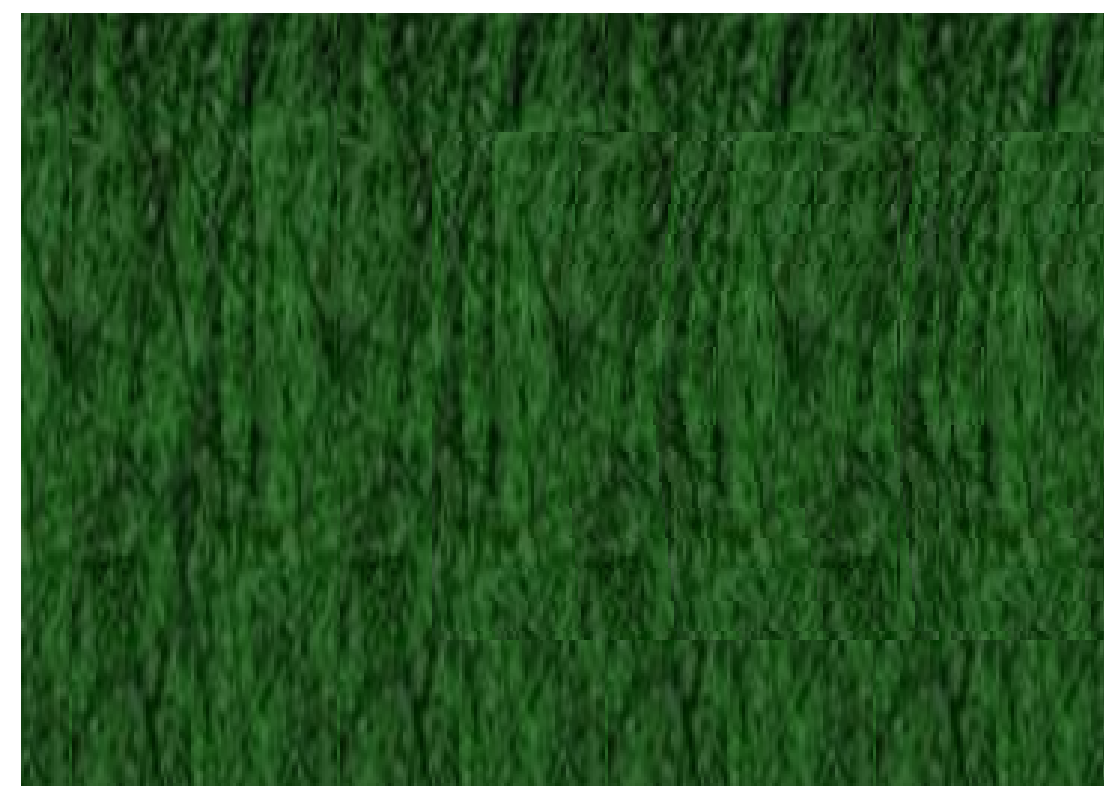

(b) pattern autostereogram

Figure 3: Examples of autostereograms 
causes binocular disparity, which in turn induces the stereo illusion. Figure 4 illustrates a red-cyan anaglyph image of Martian soil.

Additional alternatives of three dimensional viewing techniques include holograms and volumetric displays, both of which involve manipulation of light via interference, scattering or projection. These alternatives produce realistic images in 3-D; although volumetric displays are still under development ${ }^{5}$, they require specialized equipment not readily available for widespread use.

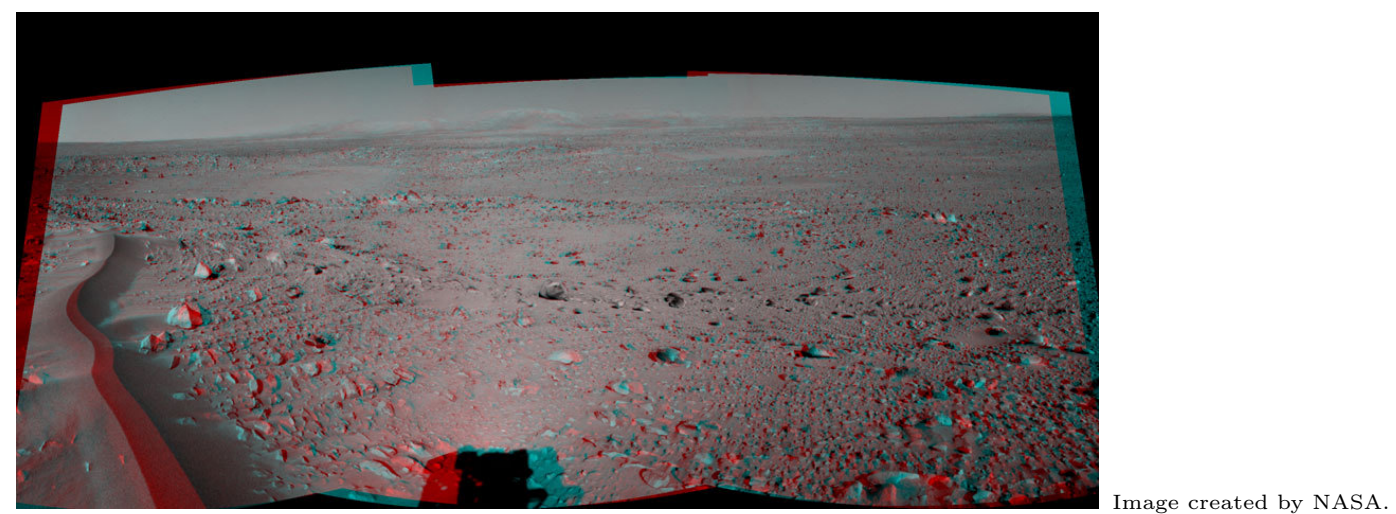

Figure 4: red-cyan anaglyph of Mars Rover

The brain's amazing ability to generate the stereo effect involves subconscious processes that coordinate eyesight. Although the physiological reason why binocular disparity produces a depth effect is beyond the scope of this document, readers interested in knowing more about it should refer to [N.Quian 1997].

\section{Depth Perception Cues}

Human beings have a natural ability to perceive depth and judge distance by various visual cues. Table 1 on page 6 describes the various depth cues of the Human Visual System (HVS).

Haze, color intensity, perspective, occlusion, shading, and blur are effects that can be easily emulated in pictures and computer screens because they can be portrayed on a flat surface. These effects are called monocular cues, which can be recognized using only one eye.

On a computer screen, we can distinguish a sphere from a circle because of the shadows and intensity. Any object represented on a flat surface will always be a two dimensional object. It is depth cues that create the illusion of a third dimension. A

\footnotetext{
${ }^{5}$ Japan's National Institute of Advanced Industrial Science and Technology (AIST) developed a 3-D projector that uses laser plasma to projects dots of light in the air
} 
Table 1: Visual depth cues

\begin{tabular}{ll}
\hline \hline Depth Cue & Description \\
\hline Haze & $\begin{array}{l}\text { Visibility of distant objects is reduced by air particles that } \\
\text { block light. }\end{array}$ \\
Intensity (Brightness) & $\begin{array}{l}\text { The color intensity of distant objects is dimmer than closer } \\
\text { ones. }\end{array}$ \\
Perspective & $\begin{array}{l}\text { Distant objects appear smaller than closer ones; parallel } \\
\text { lines tend to converge as distance augments. }\end{array}$ \\
Occlusion & Closer objects occlude those farther in the background. \\
Shading & $\begin{array}{l}\text { Appropriate shadows help distinguish relative positions of } \\
\text { nearby objects. }\end{array}$ \\
Eye focus (Accommodation) & $\begin{array}{l}\text { When focusing on an object at a fixed distance, other ob- } \\
\text { jects at different distances are blurred. } \\
\text { Etereopsis }\end{array}$ \\
& $\begin{array}{l}\text { Each eye has a slightly different vantage point that helps } \\
\text { the brain estimate distance from the angle at which the } \\
\text { eyes converge on a point of interest. }\end{array}$ \\
\hline
\end{tabular}

photograph for instance has realistic representations of these four visual queues. This is why we can easily distinguish a photograph from a drawing. Sometimes computer generated scenery may look as realistic as photographs if these visual cues are considered in detail. [Swain 1997] describes techniques on the enhancement of depth perception using monocular depth cues.

Stereopsis is strictly a binocular cue that cannot be easily represented on a flat surface or computer screen ${ }^{6}$. The reason is straightforward, there is only one image plane and all information exists at a fixed depth. Therefore, a single image will not provide different vantage points for the eyes. Furthermore, it is impossible to include binocular relief onto a 2-D image, as Leonardo Da Vinci manifested:

"It is impossible for a painting, even with extreme perfection of features, shadows, lighting, and color, to portray the same relief as that of natural objects; unless such natural relief can be seen at a long distance with a single eye. To prove it, let $a$ and $b$ be the eyes looking towards an object $c$ along the central lines $a c$ and $b c$, such that these lines coincide at point $c$; the other lines of sight lateral to these central lines can see the space $g d$ behind the object, eye $a$ can see all the space in $f d$ and eye $b$ can see all space in $g e$.

\footnotetext{
${ }^{6}$ For detailed information on the physiology of stereopsis see: Cumming, BG and DeAngelis, GC (2001) The physiology of stereopsis. Annu Rev Neurosci.24:203-38
} 


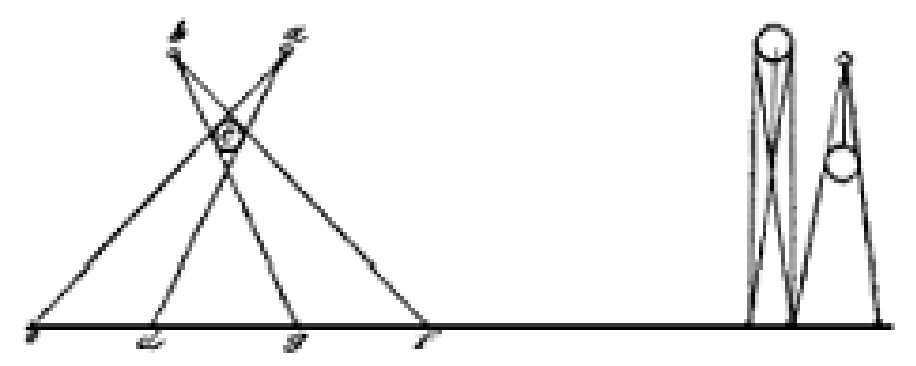

Since with both eyes we can see behind object $c$ all space $f e$, thus object $c$ becomes transparent -nothing hides behind it-; if seen with one eye this won't happen, an object larger than that eye will not be transparent, unless said object is much smaller than the pupil, as shown in the illustration. From this discussion we can conclude the following: Since any painted object occupies all space behind it, there is no possible way to observe anything on the field of view that lays behind the circumference of that object." 7

Using a stereogram we can tap into our ability to perceive depth by way of stereopsis. In section 5 we discuss a process for generating random dot autostereograms.

\section{Motivation}

A peculiarity of experiencing the random dot stereogram is that once depth perception is achieved (the stereo effect), the human visual system (HVS) is able to "lock on" to the depth at which the three dimensional (3-D) image appears. This sustained perception allows the viewer to wander the eyes through the image without losing focus on the perceived 3-D object. It is because of this "locking on" that the HVS is able to observe dynamic stereograms suitable for interactive visualization [Petz 2003].

According to [Portas 2000], this ability to remember the depth at which the object "pops up" suggests a memory-like process engaged in maintaining perceptual persistence. Using functional magnetic resonance imaging (fMRI) to measure neuronal responses on subjects experiencing random dot stereograms has suggested that memory mediates in the perceptual processing [Portas 2000]. Areas of brain activation during a 3 -D viewing experience of a stereogram include the left dorso-lateral pre-frontal cortex and the left hippocampus. Even though these brain areas associated with long term memory [Eichenbaum 1997] are involved in the process, it is debated whether their involvement is strictly to help out in deciphering the observed scene by comparing with known objects or if there is yet another process that mediates with the memory.

\footnotetext{
${ }^{7}$ Excerpt and illustration obtained from "Trattato della Pittura" by Leonardo Da Vinci, vol 2 part III entry 482. Translated from Italian by the author.
} 
From the psychological perspective, as S. Magnussen points out in [Magnussen 2001], human memory is "... not a single mental faculty or cognitive system; the cognitive process which we collectively call memory are composed of a number of independent and specialized cognitive systems that encode and store information in different formats."

The ensuing question thus: What if stereographic perspective draws upon cognitive systems to enhance the encoding and storing process of memory?

We intend to test whether information read by way of an autostereogram has any effect on memory recall when compared with regular monocular reading. Since visualizing a stereogram requires concentration, we suspect that reading a document displayed in a SIRDS may result in a more thorough and focused reading experience.

\section{Learning to see Random Dot Stereograms}

Although anyone with vision through both eyes has the ability to experience the stereograms, perceiving them for the first time may not be easy. It is difficult to "teach" how to view a stereogram since it involves converging the eyes at a distance greater or closer than that at which the image is painted (image plane). Being able to see the stereogram amounts to focusing on an object at the right distance.

Certain stereograms require that the viewer look beyond the image and focuses his/her sight to an object behind the image plane. To view the autostereograms in this project, viewers' eyes need to diverge on the image plane. Other autostereograms require the viewer to converge their eyes i.e., they need to focus on an object that is in front of the image. Figure 5 illustrates the concept of stereo disparity necessary to view stereogram.

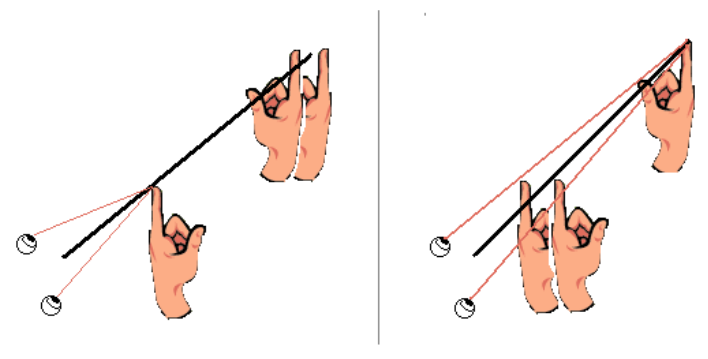

Figure 5: Illustrating stereo disparity

The pictures in Figure 5 show the stereo disparity we perceive when looking at our own hands with both eyes. Placing the left hand close to the eyes and the right hand to arm's length, we can experience stereo "double fingers". On the picture to the left, when focus is fixed on the index finger of the left hand, the right hand will appear double on the background. Similarly on the picture to the right, when focusing on the 
index finger of the right hand, the left hand will appear double on the foreground. This "double image" of the fingers is simply the information each eye receives when the focus is not placed directly on the finger; which is why the illusion disappears when one eye is closed.

As Figure 5 suggests, stereographic depth perception occurs when the eyes are focused at the correct depth. To further illustrate how to see the stereograms one needs to keep in mind that the stereo object will only appear when the "double fingers" are at a particular distance apart. Referring back to Figure 5, if we look and fix the gaze at the index finger of the right hand, the distance between the "double fingers" of the left hand increases as we move the right hand index finger away from the face. As you move the index finger back and forth you can see the disparity change between the double fingers of left hand.

Normally, stereo images "appear" at the same distance beyond the screen as the viewer's eyes are from the screen. Similar to a reflection of a person on a flat mirror, the reflection is always at the same depth as the distance from the mirror to the person. That is, if you are standing two feet away from a flat mirror, the reflection you see of yourself is also two feet behind it, thus your reflection is at four feet from your eyes. As an alternative to help viewers see the stereogram, the use of a mirror may help to focus on the correct depth.

\subsection{Instructions to see the stereogram}

At the beginning of this project we had no procedure or instructions on a way of teaching viewers how to see the stereograms. Subjects were simply instructed to look in the direction of the stereogram and relax their eyes. Using this approach was not as fruitful as expected even though some people achieved stereopsis fairly quickly (less that a minute). However most subjects found themselves looking directly at the image without being able to see the illusion. This meant that some subjects lacked the ability to diverge the eyes at will. Provided that the stereo experimentation depended upon viewers being able to learn how to see the images, a systematical description of how to view stereo images had to be provided.

As an effort to aid viewers in focusing eyes to the correct depth, we integrated two black squares at the top of the image frame. These squares are called helper dots, and are used to aid the viewer converge the eyes correctly. The use of the helper dots required that the screen be reflective enough so that it acts as a mirror and the subject can see him/herself. To use the helper dots the subject is instructed to look at the reflection of his/her nose on the screen. The reflection must occur about $5 \mathrm{~cm}$ above helper dots such that the dots can still be visible in the vicinity of the gaze. While looking at the reflection of the nose subjects can notice the helper dots converge into a solid third dot that appears in between the original helper dots. Once subjects fix their gaze at middle dot, the scene's background becomes noticeable and recognizing the $3-\mathrm{D}$ image soon 
follows.

Similar to Figure 5 on page 8 , where moving the finger changed the disparity of the foreground, the reflection prompts the viewer to look at the correct depth such that disparity is at correct distance.

Figure 6 illustrates what the viewer should see happens to the two helper dots when viewed correctly. The two dots seem to separate as the right eye focuses on the right dot and the left eye focuses on the left dot. This fusion of the two distinct images produces the illusion of a "third" dot. In order for the subjects to see the reflection of

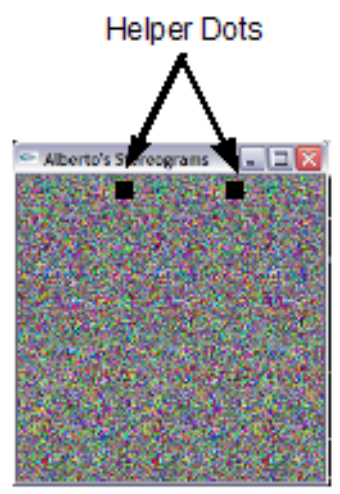

A

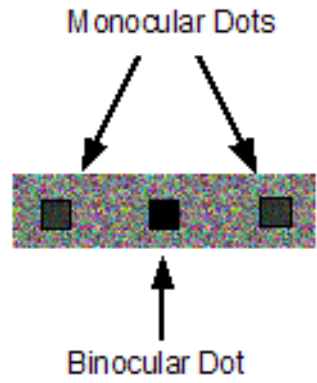

$\mathrm{B}$

Figure 6: Helper dots (stereo visual aids)

their nose on the screen a small mirror may be fixed on the screen about $5 \mathrm{~cm}$ above the helper dots. By doing this the subject is able to focus on the reflection of his/her face to converge eyes to correct depth while noticing that the dots move and converge in the periphery of the gaze. The separation of the helper dots is such that when viewed stereographically the 3-D dot converges on the far plane of the scene.

After the subject is able to see this third dot, the image stereogram image will soon follow and materialize near the center of the frame.

\subsection{Limitations of the SIRDS}

In an autostereogram, stereopsis appeals only to depth perception, therefore it is impossible to include additional visual cues onto stereographic images. With the exception of occlusion and perspective, there is no way of embedding the remaining monocular cues onto these images to make them more realistic. Even though the image that composes the stereogram may be colored randomly or texturized by repeating patterns, it is not possible to color any particular solid object portrayed in 3-D. It is very difficult, if not impossible, to represent color intensity and shadows within the stereo image. A second 
limitation, although minimized with training and practice, is the difficulty of seeing the images for the first time.

Another important limitation of the autostereogram is that of horizontal disparity itself. Since the separation of projection pixels is strictly horizontal, rotating the image plane will disturb this correspondence, hence the illusion is unattainable.

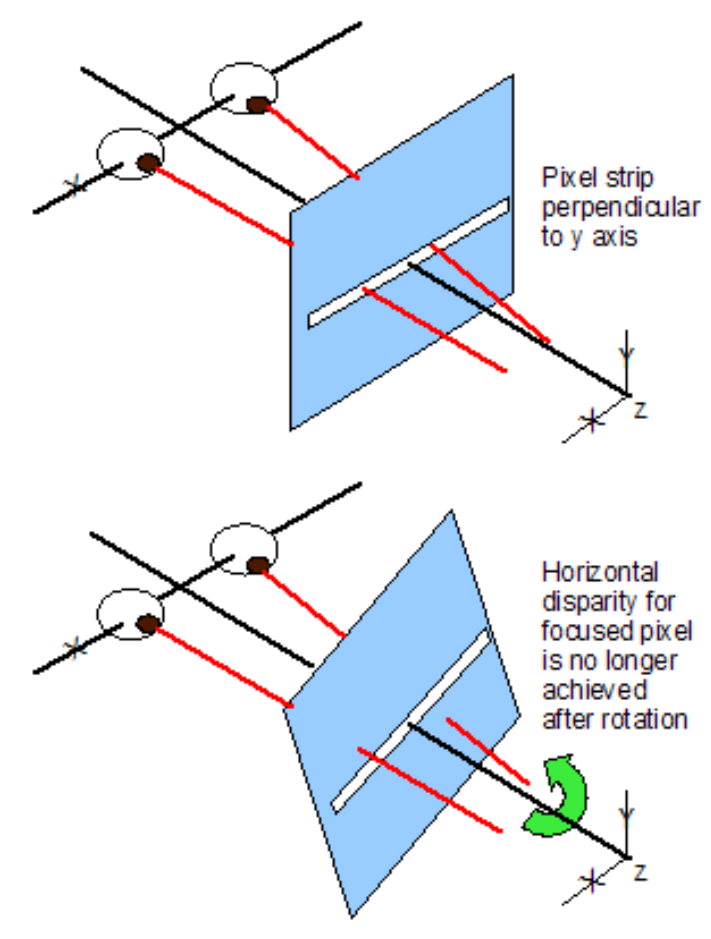

Figure 7: SIRDS horizontal disparity constraint defeated by rotation

Figure 7 illustrates why rotating the stereogram blocks the stereo illusion. Even when vergence is correct, a rotated SIRDS cannot induce depth perception. The reason is simply that there is no angular relationship between the pixels in the image plane. A rotation of the image plane will have the viewer merging two pixels that represent distinct points of the 3-D object. Since there is no relation between these points depth perception cannot ensue. The red lines denote the pixels that both eyes focus after rotating the image plane, notice that red lines now point to different pixels that are unrelated. Although rotation of the image plane interferes with pixel correspondence, it is possible to rotate the image slightly and still preserve the illusion. The eyes can still focus a SIRDS rotated approximately 25 degrees to the horizontal while maintaining the stereo effect. This capability is due to the ability of the eyes to rotate slightly about their axes. 


\section{Creating the Autostereogram}

In this section we discuss the mathematical details behind the creation of the autostereogram. The idea behind perceiving an ordinary 3-D object on a 2-D image is that for every pixel in the object's surface there should be exactly two corresponding projection pixels in the image. These two projection pixels will correspond to the two vantage points from left and right eyes. See Figure 8 for a geometric explanation. The horizontal disparity between these projected pixels triggers the brain into reproducing the stereo effect necessary to view the 3 -D object. The ability to use both eyes to produce depth perception is called stereopsis.
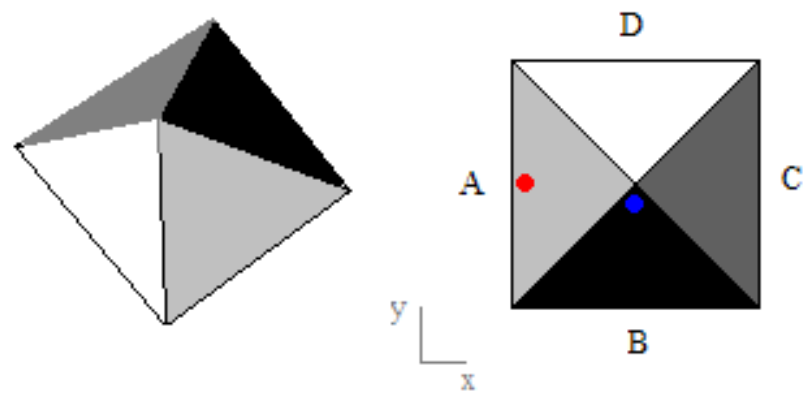

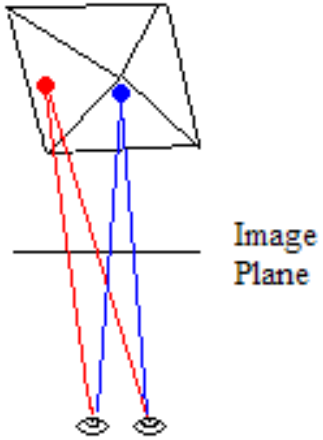

Figure 8: Vantage points on a pyramid that point to the viewer

In Figure 8 the pixels near the base of a pyramid (red dot) are farther from the viewer than the pixels near the apex (blue dot). As stated above, for each pixel in the surface of the pyramid there should be two corresponding pixels for the left and right eye projections. The image plane, represented by the black horizontal line mid-distance between the object's base and the viewer's eyes contains the four pixels that represent the blue and red pixels on the pyramid's surface. The points where the red and blue lines intersect the image plane represent the relative positions of the projected pixels. The horizontal separation of these pixels conveys the depth information to the eyes. In this example, since the blue pixel is near the apex, the separation between the blue rays are closer together that those corresponding to the red pixel. Projected pixel separation is directly proportional to the depth of the pixel in the 3-D object's surface.

\subsection{Calculation for Projection Pixels}

Converting a 3-D object into an autostereogram requires a projection transformation of every pixel of the object's surface. Figure 9 (adaptation from [Thimbleby et al. 1994]) 
illustrates a geometrical model on how to derive the transformation formula, where $\mathrm{E}$ is the distance between the eyes, $\mathrm{S}$ the linear distance between the projection of a surface pixel at a depth of $z$. The depth value $z$ is always within the field of depth, which in effect is the range of depth at which we can portray 3-D objects. A value of $z=0$ locates a pixel on the autostereogram's background while a values of $z=1$ denotes the closest pixel portrayed in the viewing volume.

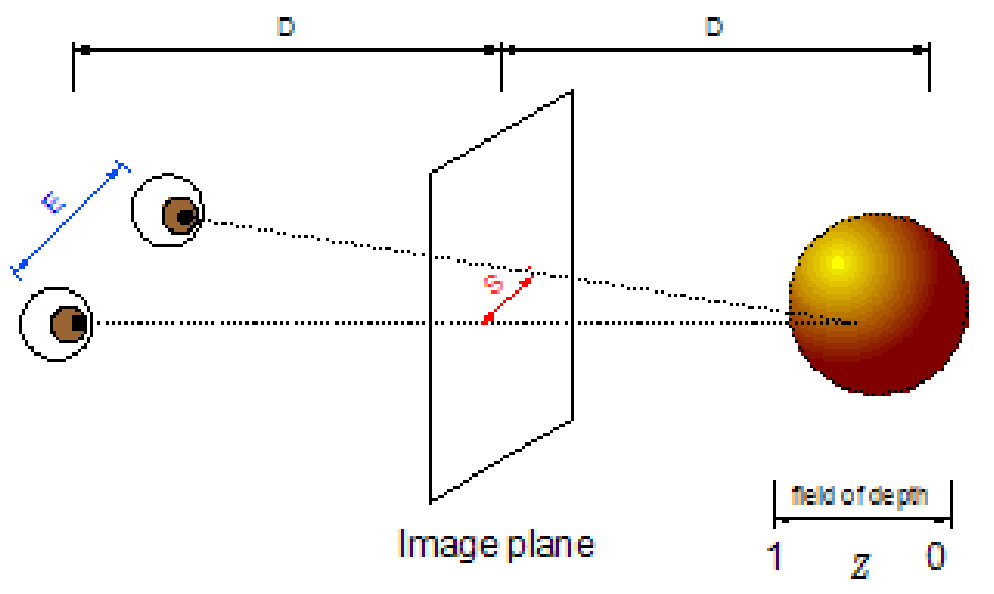

Figure 9: Geometrical projection model

The pixel separation formula we use for generating the autostereogram was obtained from [Thimbleby et al. 1994]. Let us consider that the viewer is at a distance $D$ from the image plane. The autostereogram's background is chosen to be at the same distance behind the image plane as the viewer is in front of it (recall the flat mirror reflection relation on section 4), therefore we have that the viewer's eyes are at a distance of $D+D$ from the background. The units for all measurements will be expressed in terms of screen pixels.

Let the distance between the eyes be E, which is roughly 230 pixels $(2.5 \mathrm{in} * 92$ pixels/in). We must now select the space that can be portrayed beyond the image plane. The farthest a pixel can reside within the portrayable space is at the background, namely the far plane; it is represented with the depth value $z=0$. The nearest portrayable pixel can occur when $z=1$ and it is called the near plane. The field of view (FOV) is precisely the space between the far and the near plane, i.e. the portrayable 3-D space. Although this field of view can be chosen to be the entire space $D$ behind the image plane, it is hardly done since it would be impossible to focus on the 3-D object comfortably. Using all space $D$ as the field of view would require significant vergence between near and far surfaces resulting in a $3-\mathrm{D}$ object difficult to visualize. Thimbleby et. al. use the FOV as a fraction $\mu$ of $D$, particularly $\frac{1}{3}$. 
On the one hand, an appropriate thickness of the FOV allows for an easier focus on the stereo object; on the other hand, a FOV too narrow will defeat the purpose of depth perception causing the 3 -D object to appear nearly flat.

The formal derivation is as follows:

Since the triangle delineated by left eye, right eye and 3-D pixel is similar to the triangle delineated by the left eye projection, right eye projection and 3-D pixel, we have

$$
\begin{aligned}
\frac{E}{S} & =\frac{D+D-D \mu z}{D-D \mu z} \quad \text { by Similar Triangles } \\
& =\frac{D(2-\mu z)}{D(1-\mu z)} \\
S & =\frac{1-\mu z}{2-\mu z} E
\end{aligned}
$$

where $\mu$ is the fraction for the FOV and $z$ is the pixel's depth.

\subsection{Pixel color constraints}

Any single image random dot stereogram can be created with as few as two distinct colors. The only color constraint imposed is that both left and right image projection pixels from any 3-D point on a surface must have the same color. Sometimes it may occur that two distinct pixels on the 3-D surface happen to project on the same point on the image plane, see Figure 10.

When any projection of two pixels coincide on a location on the image plane, the same color must be used for both projected pixels. In Figure 10 the right pixel projection point for pixel $\mathrm{A}$ is the same as the left pixel projection point for pixel $\mathrm{B}$, therefore the three locations pointed to by the black arrows must be colored equally. This condition must be upheld provided A (B) does not occlude B (A). Section 5.3.2 describes artifacts that may occur when this condition is not met. Depth perception on an autostereogram is entirely dependent on preserving similarities between pixel pairs that represent each 3-D point on the surface.

\subsection{Program implementation issues}

[Thimbleby et al. 1994] discusses an implementation that uses depth map information to construct the stereographic objects. This depth information is generally a grayscale 


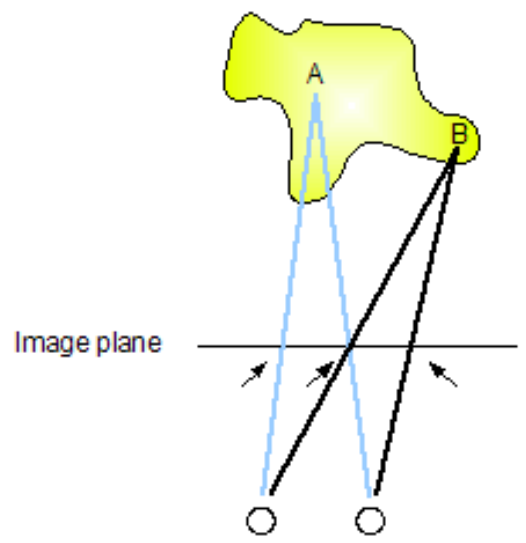

Figure 10: Shared projection points on image plane

image whose values represent the relative distance from the object's surface to the viewer. Lighter shades of gray usually represent surfaces closer to the viewer while darker areas denote objects farther from viewer. The generation of the depth map is usually a pre-process that then serves as the input for the stereogram generator.

Our algorithm makes use of the graphics hardware to generate the depth information in order to accelerate the rendering process. The depth map for any scene is produced by means of the z-buffer available through the Open Graphics Library (OpenGL). The advantage of using the z-buffer is that the depth information of any pixel in the surface can be generated automatically by the hardware; these values are stored in the graphics hardware memory for easy retrieval and higher performance. Refer to Appendix A for code listing.

\subsubsection{Flat layers in the autostereogram (depth steps)}

On lines 9-13 from the listing file randompattern.cpp we notice the separation formula macro. It is important to note that the output of separation is a step function in terms of its output. For distinct values of $Z$, separation( $Z$ ) may yield same separation distances. For example, both separation (0.47) and separation(0.51) have the same output value of 82 . Table 2 illustrates how distance values are mapped according to pixel depth while using a field of view of $\frac{D}{3}$ and a screen resolution of 72 DPI.

As shown in Table 2 there is no one-to-one correspondence between the pixel depth and the separation distance, thus, there will be no smooth transition across the different depths of the autostereogram's surface. This is the reason why we see stereo objects seem to be made of stacked layers of flat panels. The largest separation distance for two projection pixels corresponds to separation(0), namely the far plane. Conversely, 
Table 2: Mapping pixel depth to projection separation

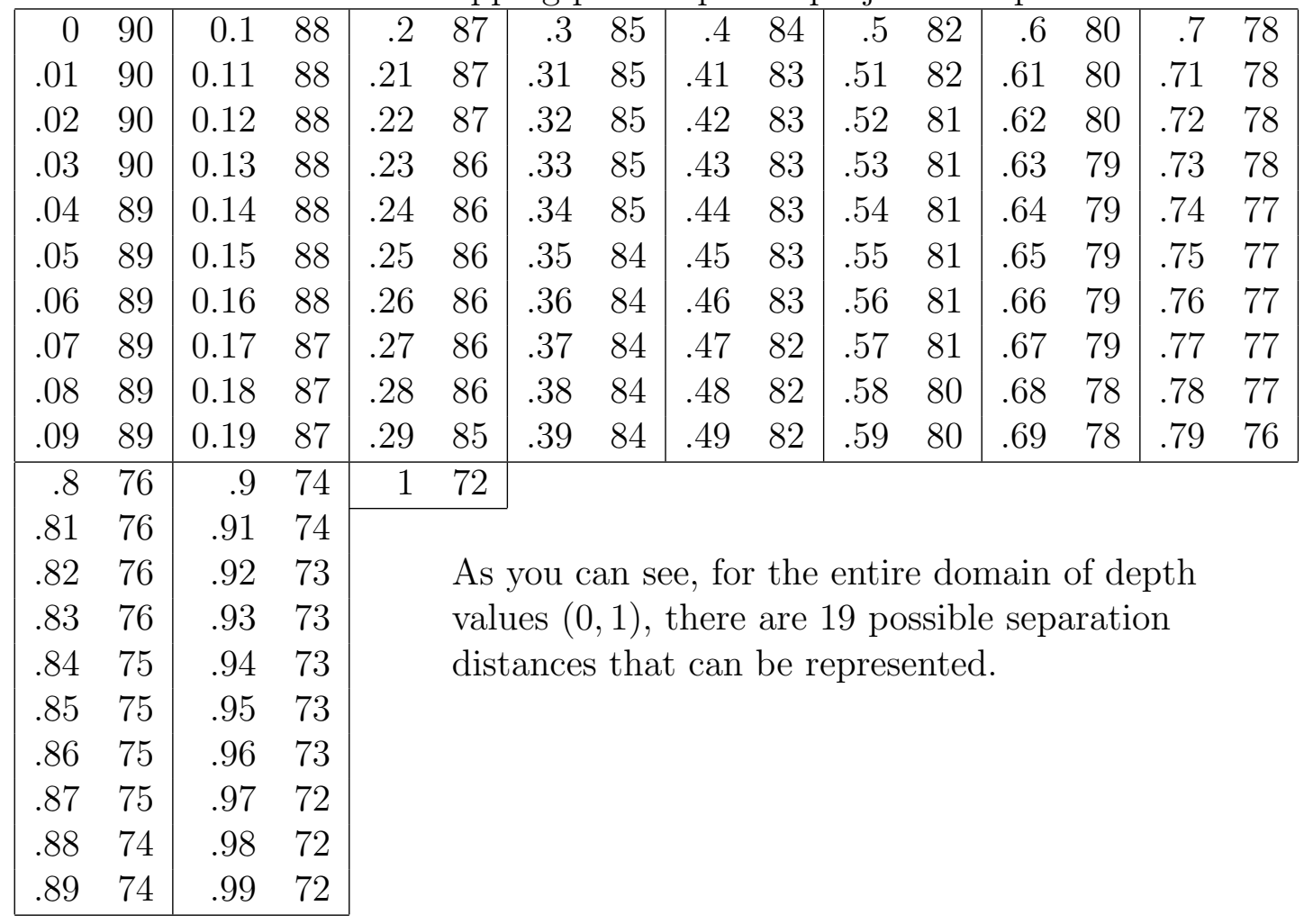

separation(1) corresponds to the near plane. The distance number is always an integer since pixels cannot be separated by a fractional amount.

To eliminate this "layered" appearance a new mapping function needs to be devised such that there exists a one-to-one correspondence between pixel depth and projection separation. Removing the visible gaps amounts to rewriting the separation formula into a 1-1 function. ${ }^{8}$ The immediate problem of discovering such function is that it would require a display with infinitely fine resolution.

Given that the layer problem cannot be eliminated because of the inconvenience mentioned above, there is a way to effectively reduce the visibility of such gaps. One way to reduce the layered appearance is to increase the resolution of the image plane. Finer resolution will allow a larger number of planes constituting the stereo object. Increasing the density of such contiguous planes causes the illusion of a smooth 3-D surface. In the absence of a finer resolution medium, increasing the resolution programmatically is another option; instead of having 72 or 92 dots per inch (DPI) a larger DPI achieves

\footnotetext{
${ }^{8} \mathrm{~A}$ one-to-one function is a function for which every element of the range of that function corresponds to exactly one element of the domain. In this case, the range of separation( $\mathrm{X}$ ) is the 3-D depth value and the domain is the projected pixel separation.
} 
the same effect of increasing the image planes, however the resulting stereo image would require the viewer to orient eyes at uncomfortable degrees of vergence.

A simple solution that does not involve increasing the resolution consists of varying the pixel separation distance across the horizontal lines. The snippet of pseudo-code below illustrates how this variation is achieved. In the code, mask [i] represents an array that stores the depth information for the pixels across a horizontal line, i.e. a scan line. For each pixel in the scan line we compute its horizontal separation; on even scan lines this separation is unaltered, on odd scan lines the separation is randomly modified by 1 pixel to the right or 1 pixel to the left. This alteration creates a blending effect that generates continuity across the 3 -D surface.

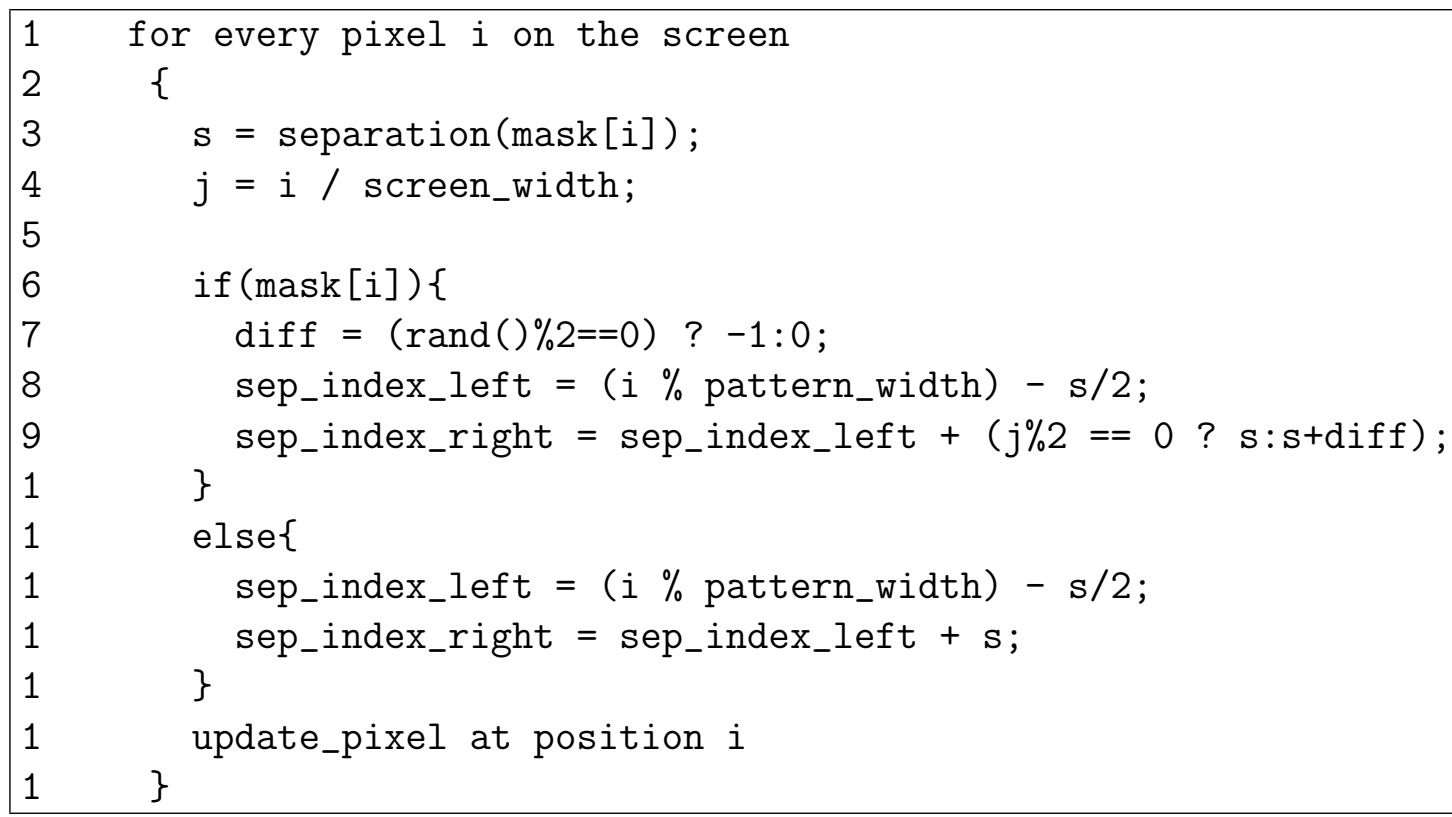

Figure 11 on page 18 illustrates how the pseudo code above generates an autostereogram with a fairly homogeneous 3-D surface.

Another popular method to improve the appearance of the autostereogram is to make use of a background pattern. Instead of having random dots conveying depth perception, each pixel on an image can be modifies to achieve the same effect. 


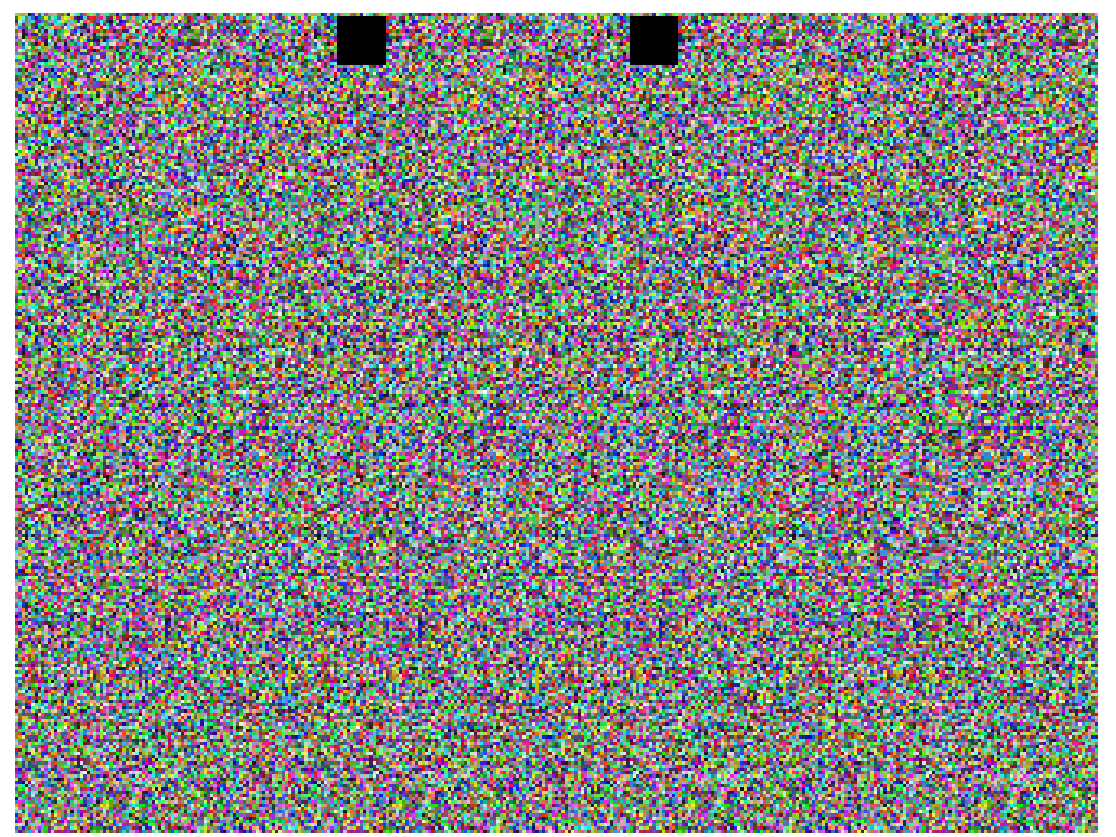

(a) flat depth

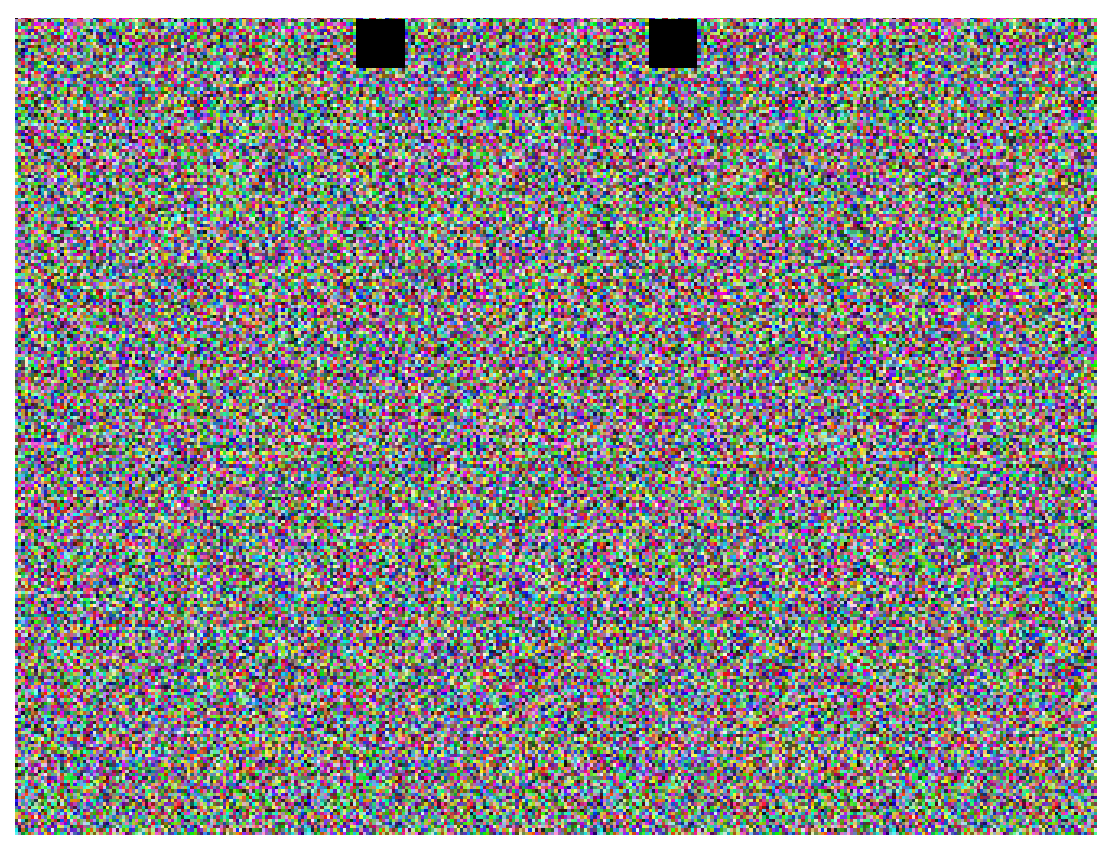

(b) homogeneous depth transition

Figure 11: Improving 3-D surface homogeneity 


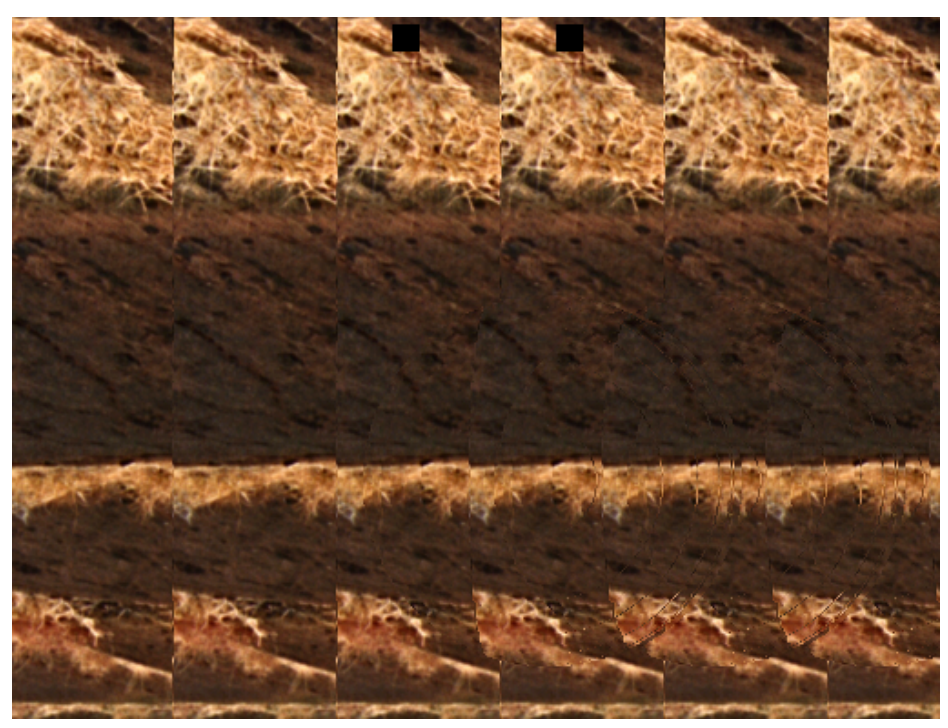

\subsubsection{Avoiding 3-D artifacts}

If pixel projections are not constrained properly, corresponding pixels at different depths would cause the production of objects that are not part of the original geometry of the 3D figure. For instance, suppose a surface pixel at location $P$ has projections $P_{L}$ and $P_{R}$. Suppose also that a pixel $Q$ lays on the background. If $Q_{L}$ happens to be the projection location of a previous pixel whose depth value was greater than that of $Q$, then an artifact is mistakenly generated when $Q_{R}$ is set the same color as $P_{R}$. See accompanying illustration in figure 12(a). For this reason we must check under which circumstances two pixels are allowed to be the same color. Our routine to generate the autostereograms processes the pixels from left to right, from this we know that location $P_{R}$ is already associated with pixel $P$ when $Q_{L}$ attempts to use the color already mirrored from $P_{L}$. Figures $12(\mathrm{a})$ and (b) demonstrates an example of the artifacts that occur inside a 3-D ring.

\subsubsection{Custom font set}

One of the most challenging aspects of this project was discovering a font configuration appropriate for comfortable reading. It was essential to create a set of fonts easy to read and simple enough not to be distracting. Correct character size, shape, 3-D thickness and width are the characteristics that make a font readable in a stereographic context.

Given that there is no contrast between the random dot image in the stereogram and the text within it, character size need be large enough; otherwise all letters would be camouflaged against the background. Since each letter is only visible by the effect of its depth, it is necessary to represent bulky three dimensional text. Figure 13 illustrates how the characters are modified to be visible within the stereogram. The goal for 


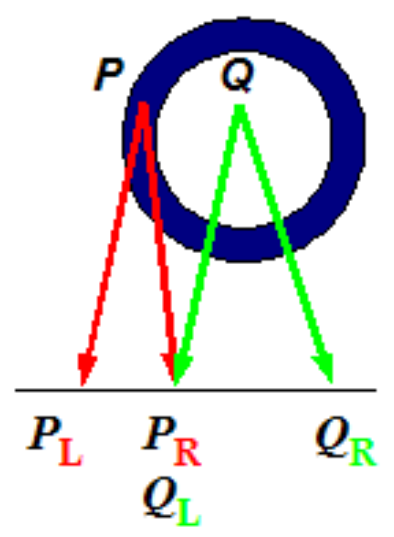

(a)

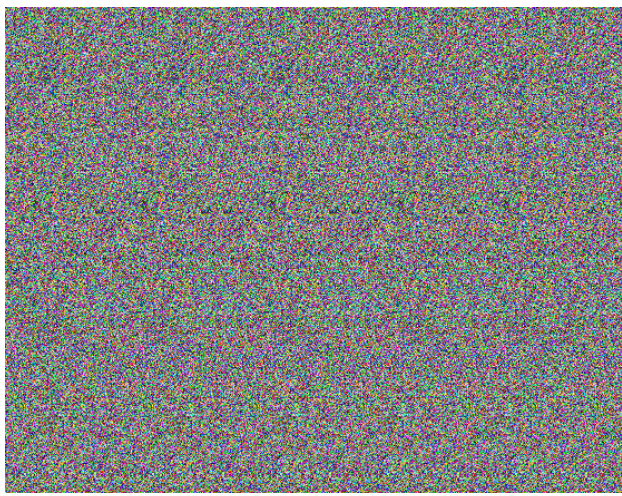

(b) Artifacts form inside ring

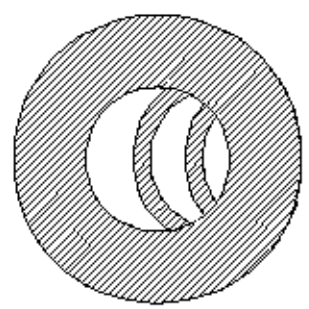

(d) Corresponding Monocular views of figures (b) and (c)

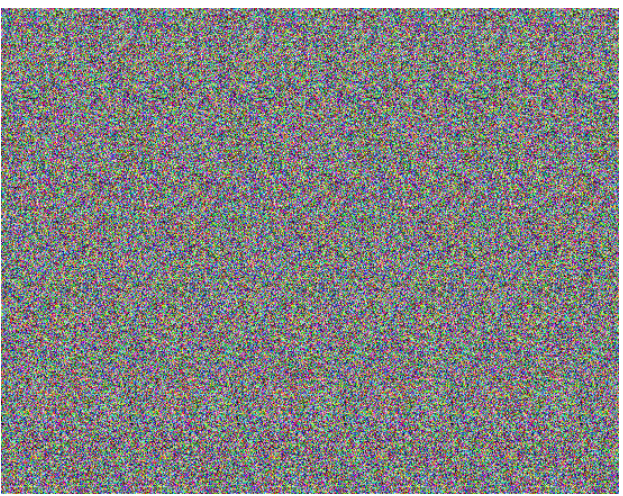

(c) Area inside ring free from artifacts

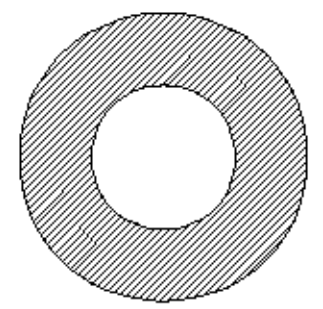

Figure 12: Avoiding artifacts from forming in 3-D surface 
an appropriate font is to be large enough to represent 3-D perspective while avoiding being too large. Figure 14 shows the alphabet of custom characters displayed in their monocular version.

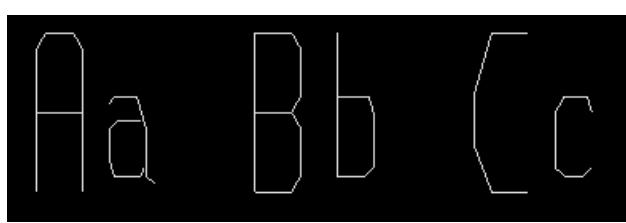

(a) original

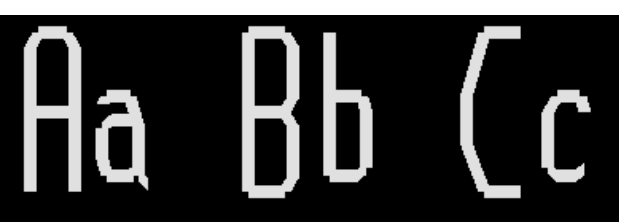

(b) wider

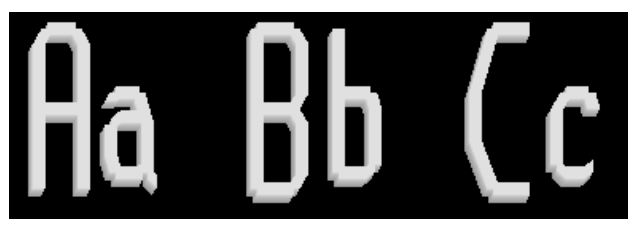

(c) 3-D thickness

Figure 13: Adjusting the 3-D fonts

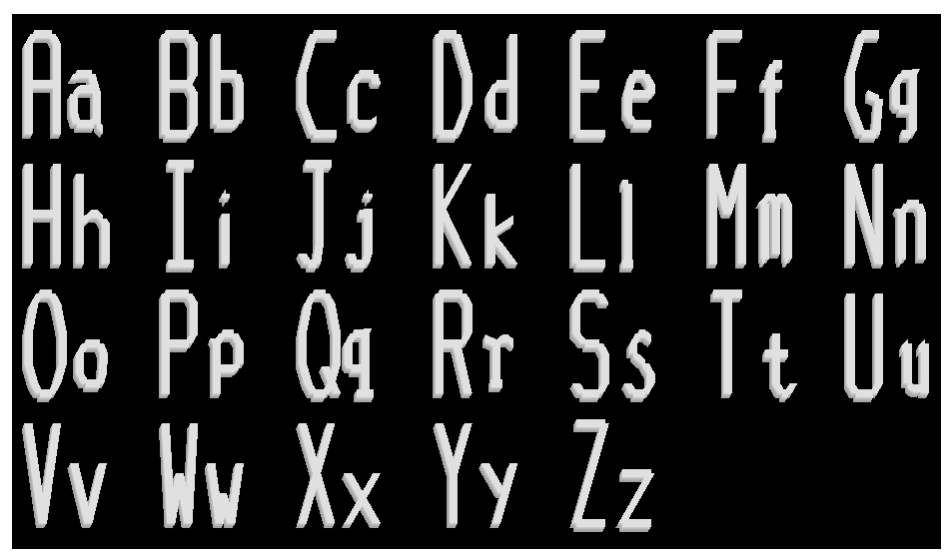

Figure 14: Font alphabet

There exists a compromise between font size and visibility that we should mediate. It is known that larger wider fonts are easier to read in the stereogram; the problem is that only few words would fit in the width of the screen. There is a trade-off between the amount of text that can fit in a single line and the size of the characters in the text. Our objective is to represent stereographic text as close as possible to its monocular representation. Currently we can fit five to six stereographic words per line on a window 1018 pixels wide. More words per line would involve reducing the size of the characters, therefore increasing the difficulty of recognizing the characters in words. 


\subsection{Software functionality}

The autostereogram generator we developed for this project contains a limited graphical user interface that allows for the variation of the following parameters:

\section{character width color \\ random dot granularity helper dots background pattern}

As per the information learned in section 5.3.3, font depth and thickness parameters have been adjusted to what we believe are comfortable viewing settings and are not modifiable at real time. Character width can be modified at will to help the user in the event that a wider text is preferred. Color and granularity are used as an alternative to test on the ease of comfort for the viewing experience. For some users, the black and white stereogram is easier to distinguish as opposed to a random colored version. With variable granularity, the size of the pixels that compose the image are also variable; coarser random dots tend to have less resolution but are easier for the unexperienced viewer to see. The helper dots is an extremely important feature to help first time viewers focus eyes correctly. The helper dots can be toggled on or off. The background pattern is an additional alternative to test contrast comfort.

\section{Previous Work}

Even though the stereogram is a commonly used tool in Art [Morland 1976], Neurophysiology [Julesz 1972], Visualization [Petz 2003][Schroeder et al. 1998] and Psychology its potential use has not been extensively explored. SIRDS have been used to measure thresholds of human cognition[Nakagawa 2005], even depth perception in infants; but no explicit research on the effects they may have on memory. Although autostereograms have been used on experiments to test for eidetic imagery [Crawford et. al.], their use did not suggest effects on memory, it was merely used as a tool to present information in partial form.

\section{Method}

To test whether or not there is an effect on immediate memory recall after information is experienced by way of an autostereogram, we devised a control group study with a simple reading experiment. Using the autostereogram application, we allow two groups of people to read a story either in monocular or stereographic fashion. Group 'Monocular' -monocular readers- will read the story in the "normal" way while group 'Stereo' -stereo readers- will read the story stereographically. 
The fictional story presented contains details of names, locations, dates and events. Immediately after the reading experience, information recalled by subjects is measured by way of a questionnaire. This questionnaire contains a total of nine questions, including multiple choice and fill in the blanks.

\subsection{Target story and questionnaire}

The story itself is not meant to be a literary masterpiece, it is merely a collection of details in a somewhat coherent storyline. The main idea is to provide an original set of details that have no direct correlation to anything known by the subject. Refer to Appendix $\mathrm{C}$ to see the story and the questionnaire used in this study.

\subsubsection{Nature of the questions}

The choice of questions in the questionnaire is our attempt to quantitatively and qualitatively categorize differences between both types of reading.

For quantitative assessment, questions 2-6 provide multiple choice answers in which "none of the above" is among the alternatives, and only a single answer is correct. Questions 1, 7 and 8 allowed the subject to provide a written response. We used these three questions to qualitative asses memory recall.

Although question 2 asks for a date, it involves both memory and attention to detail. The story only mentions the birth year of one character, from this and other information, the subject must deduce the birth year of the other character.

For this experiment we expect that there will be a difference in information recall between both groups, with the stereo group obtaining higher scores.

\subsection{Procedure}

Subjects on this study were mostly college students who voluntarily consented to participate in the experiment. No special considerations were taken when selecting subjects. It is important to note that all experiments were performed in an outdoor environment with no control of extraneous events. Subjects viewed the images and read the story on a portable computer. They were not allowed to read the story out loud to avoid potential auditory memory encoding that may enhance retention.

Subjects were randomly selected to belong either in group 'Stereo' or 'Monocular'. When selecting people in group 'Monocular' it was not a requirement for them to be able to view the stereograms during the alloted time of the experiment. On the other hand, participants of group 'Stereo' necessarily had to be able to view the stereo images.

Participants were initially presented with stereograms depicting various geometrical 3-D figures which they must identify. The purpose of this step is to allow the subject to get a feel on how to look into the screen to produce the desired depth convergence. 
At this point, if the subject has trouble perceiving the figures, we go over the training procedure (explained in section 4) and allow him/her to relax. It is important that the subject does not feel any pressure when asked to see the images, for we believe this will compromise his/her confidence, thwarting 3-D perception. Subjects are asked to relax and take their time.

In the event that the subjects take longer than 15 minutes to see the initial images, the stereo test is suspended. The amount of time necessary to achieve correct vergence in beyond our control, therefore we only test individuals who can perceive the images in relatively short time. Once subjects correctly identify the hidden figures we proceed to show the stereo alphabet. There are two reasons for showing the stereo alphabet, first it allows the subject to familiarize with the shape of the letters, and second it is an opportunity to toggle the appearance of the autostereogram (as discussed in section 5.4) to allow the viewer to choose the most comfortable properties.

Participants are reminded that the story requires them to scroll the page with the up-down arrow keys, and that it may be convenient to keep the fingers over these keys to avoid removing the eyes from the autostereogram to look at the keyboard.

After subject completes reading the story the questionnaire is administered immediately.

\section{3 how long does it take to see the illusion?}

During the implementation phase of this project voluntary participants viewed the images to test the depth effect. The time elapsed for stereo perception on subject who had never seen stereograms ranged from less that 10 seconds to a few hours.

\section{Results}

A total of 30 people participated in this study, 15 on each group. Of the 15 people on each group there were 7 female and 8 male participants.

Results were tabulated according to correct or incorrect responses, blank questions were disregarded for both groups. On written responses, correct answers were credited only when exact answer was provided or when the answer contained at most one misspelled character. Each questionnaire contains 8 questions pertaining to the story itself, and an additional multiple choice question that categorizes the comfort level while reading the autostereogram.

Table 3 shows the distribution of correct and incorrect responses for both test groups. The overall ratio of correct to incorrect responses is very similar between stereo and monocular readers, $62: 42 \approx 1.48$ and $66: 48 \approx 1.38$ respectively. 
Table 3: Questionnaire answer tabulation

\begin{tabular}{|c|c|c|c|c|}
\hline \multicolumn{5}{|c|}{ Group answers } \\
\hline \hline Question & \multicolumn{2}{|c|}{ Stereo Readers } & \multicolumn{2}{c|}{ Monocular Readers } \\
& Correct & Incorrect & Correct & Incorrect \\
1 & 7 & 6 & 7 & 8 \\
2 & 5 & 10 & 7 & 8 \\
3 & 10 & 5 & 11 & 3 \\
4 & 7 & 8 & 4 & 11 \\
5 & 4 & 9 & 7 & 7 \\
6 & 13 & 2 & 14 & 1 \\
7 & 3 & 0 & 5 & 8 \\
8 & 13 & 2 & 11 & 2 \\
\hline totals & $\mathbf{6 2}$ & $\mathbf{4 2}$ & $\mathbf{6 6}$ & $\mathbf{4 8}$ \\
\hline
\end{tabular}

Stereo and monocular reading response comparison

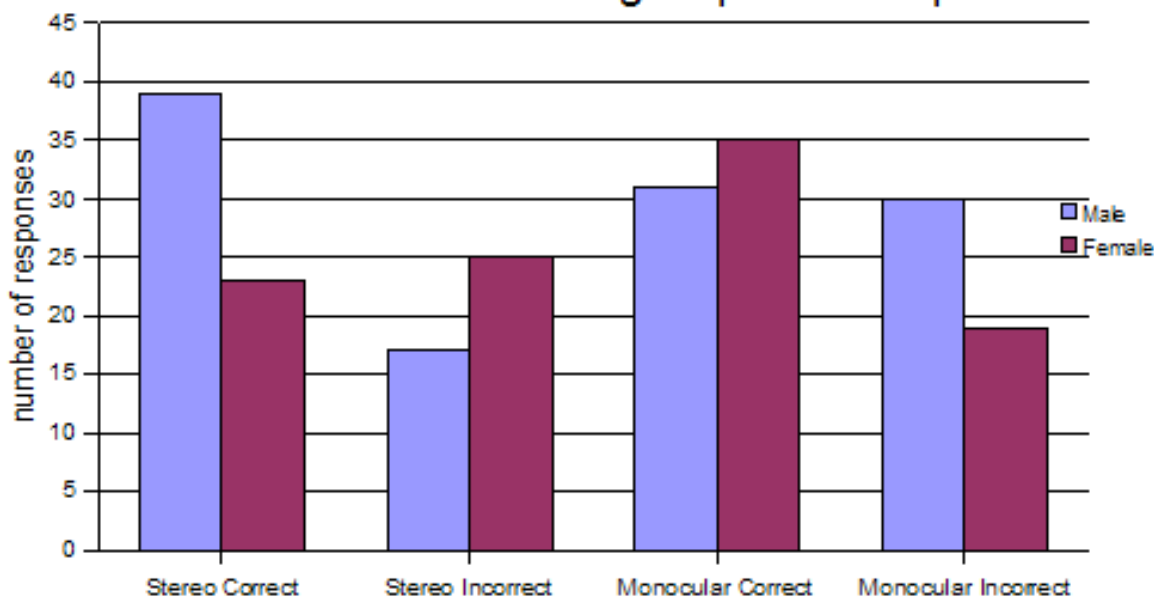

The graph above illustrates the score distributions for males and females on both 'Stereo' and 'Monocular' groups. 


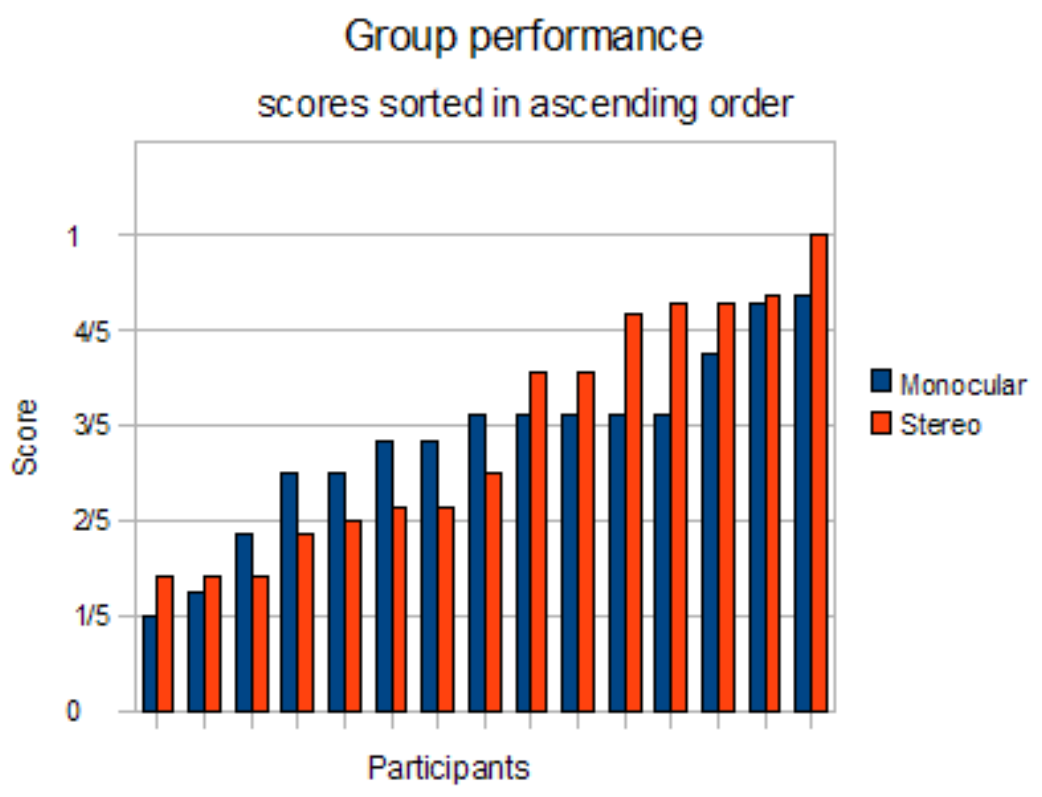

Table 4: Group score statistics

\begin{tabular}{|c|c|c|}
\hline & Monocular & Stereographic \\
\hline & & $\frac{3}{7}$ \\
Score Mean & $\frac{4}{7}$ & 0.5 \\
Score Median & 0.63 & 0.25 \\
Standard Deviation & 0.19 & \\
\hline
\end{tabular}

Table 5: Comfort level distribution for stereographic readers

\begin{tabular}{|c|c|}
\hline Total discomfort & $0 \%$ \\
Partial discomfort & $57 \%$ \\
Partially comfortable & $36 \%$ \\
Highly comfortable & $7 \%$ \\
\hline Correlation coefficient & \\
relating score and comfort level & 0.57 \\
\hline
\end{tabular}



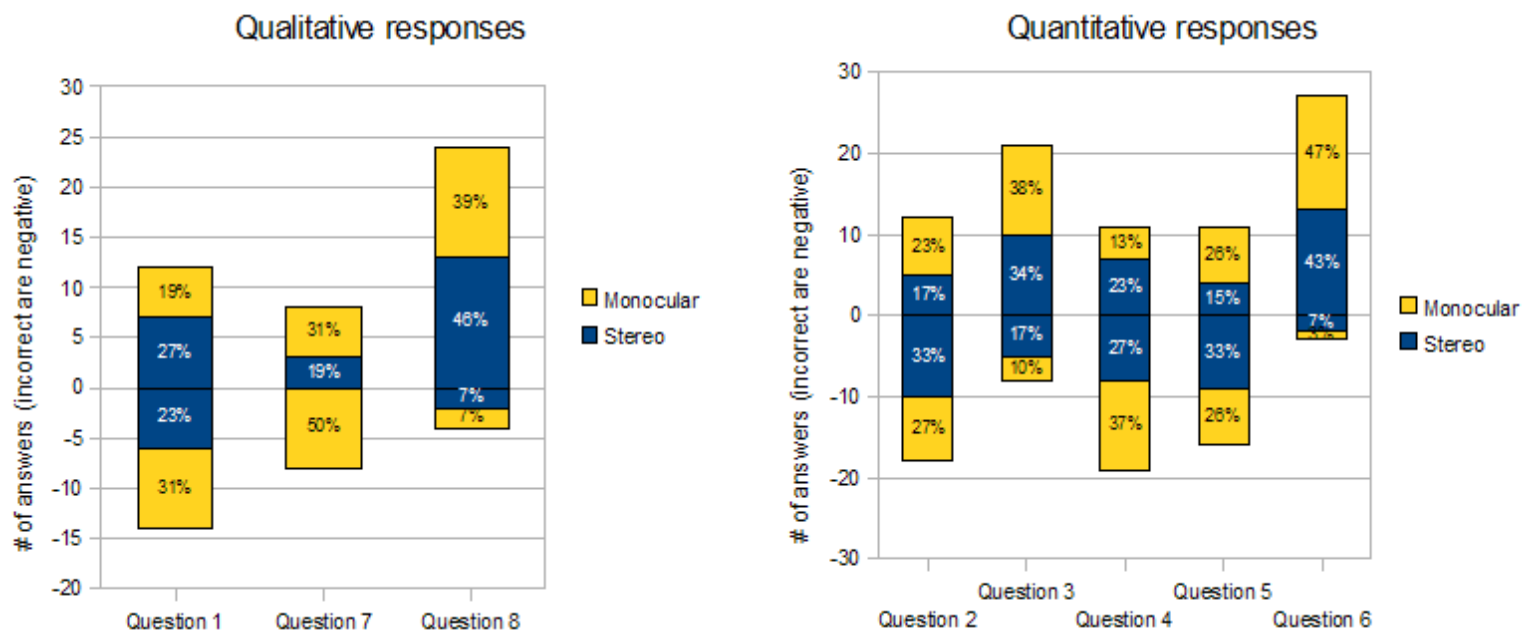

Question 1 on "Qualitative responses" graph contains 12 correct monocular responses instead of the 14 correct responses reported in table 3, this is because two participants only checked the correct choice provided but did not write the answer.

\section{Discussion}

Although the ratios of correct to incorrect answers are very similar in both 'Stereo' and 'Monocular' groups, there is a slight advantage on the performance of stereo readers. As the measures of location on the data suggest (Table 4), memory performance for stereographic readers is no worse that monocular readers. Even though the median for stereo readers is smaller that those from the monocular group, from the Group performance graph we can notice that higher scores belong to the stereo group. Median measurement is insensitive to smallest and largest magnitudes at both ends of the score spectrum. However, we can see that the standard deviation (std) is greater for the stereographic group (std monocular group 0.19, std stereo group 0.25). Given that both groups have a similar mean value, 0.57 and 0.60 for monocular and stereo groups respectively, the variability suggests that higher scores are more likely to occur on stereo readers. From graph "Stereo and monocular reading response comparison" we can notice that female readers performed better than male subjects by $6 \%$ on the monocular group. Conversely, stereographic male readers out performed their female counterparts by $25 \%$.

The Qualitative and Quantitative graphs illustrate that questionnaires from stereographic readers predominantly contain less incorrect responses than those from monocular readers. From this data it is readily seen that questions 6 and 8 are most frequently answered correctly on both groups. The prevalence of correctly answering question 8 provides evidence to corroborate the fact that repetition plays an important part in memory recall; for question 8 inquires about the pet's name in the story, which is mentioned three times throughout the story. As for question 6 , inquiring about the time it 
took the main character of the story to roast a chicken, it can be understood that it is mostly answered correctly for two mutually exclusive reasons. Either the reader is aware of the long time it takes to roast food, thus remembering the fact from the story; or the reader considers the length of time bizarre and is able to remember this information because of its unusualness.

\subsection{Notes during the experiments}

While learning to diverge eyes at appropriate depth, viewers have difficulty transitioning from the helper dots to the middle of the screen. This difficulty can be attributed to the accommodation and convergence relation ${ }^{9}$ of the HVS. When looking at a stereogram a dissociation of vergence and accommodation produces visual fatigue.[Emoto et al. 2005]

Subjects who could not see the stereo images commented on their long-lived inability to see these types of images. Regardless of our efforts to help them focus on the images, some of these participants remained convinced of their inability to see the stereo figures; which would predispose them not to succeed with the illusion. Nevertheless, of the inexperienced viewers that were able to perceive the illusion, subsequent images become easier for them to see. This demonstrates that a person can learn how to experience the depth effect in short time.

During the presentation of the 3-D figures subjects demonstrate enthusiastic interest on the stereographic objects and seem to enjoy seeing them. However, the same level of enjoyment is not apparent during reading text. Judging by the reaction of subjects, viewing the stereograms for the first time produces mild excitement accompanied by a smile or a chuckle.

While selecting the background image there is a preference for the black and white random dots. We speculate that random colors may be distracting to the viewers because in both cases the information and the depth of focus also remains unaltered.

\subsection{Memory recall versus level of comfort}

As table 5 shows, there is a positive correlation between performance and level of comfort. A correlation coefficient of .57 suggests that higher reading comfort would increase scores. Experienced participants that had seen stereo images before achieved stereopsis immediately and it was not too difficult for them to read the story.

We argue that information stored in a stereogram will be preserved in short term memory better than information obtained by normal monocular reading for various reasons:

\footnotetext{
${ }^{9}$ While looking at distant objects the angle between the axes of sight increases slightly; while this happens(convergence), the ciliary muscles adjusts the lens to focus on deep objects(accommodation). Conversely, the opposite happens when focusing on a closer object, the angle between axes of sight decrease and lenses adjust focus closer.
} 


\section{- Stereographic readers read slower}

Making out the words in the autostereogram is by itself a visual challenge because the eyes are not accustomed to reading stereographically. This difficulty necessarily forces the reader to read slower and look more carefully at the words, which avoids the repercussions of fast-paced reading that may lead to lack of attention to important ideas. Readers of the "normal" text may skim over important details. By the same token, it may be argued that slower stereographic readers are at disadvantage of forgetting earlier details read; opposed to monocular readers who finish the story faster and complete the survey with a fresh memory of all details. However, there is no evidence that suggest stereo readers are worse at remembering earlier details of the story.

\section{- Additional concentration necessary to interpret message}

Paying undivided attention to reading is undoubtedly beneficial to understanding what is being read. An interruption in concentration while reading an autostereogram often leads to loosing the depth effect, consequently dissolving the stereo text. For this reason the viewer rather keep looking at fixed depth in order to avoid having to readjust sight once they look away.

\section{- Stereo reading may be more appealing to experienced viewer.}

Interest and motivation are among the most important aspects of the readingthinking process. Reading the stereographic selection compares to discovering a hidden message; which by itself may provide sufficient motivation to keep the reader engaged, moreover, interest may stem from a pleasant reading experience when characters look appealing.

It goes without saying that if a particular topic is not of interest to a person it is impossible to expect and engaging reading experience. The perception process may also be defeated by discomfort when the stereographic text is difficult or uncomfortable for the viewer to see. More than half of the participants (57\%) considered that reading the stereographic text was uncomfortable. This claim is more prevalent with subjects inexperienced with looking at stereograms.

Although comfort level depends upon subject's ability to diverge the eyes, increasing display resolution may improve the contrast between the characters and the background. Improving stereographic text perception may consequently improve the reading experience, hence improving memory recall.

\section{Final Remarks}

There exist a small difference between perceiving information stereographically and monocularly, the former representing an approximate $3 \%$ improvement in terms of short 
term memory recall. We attribute this difference to a reader that may be paying more attention to the stereo version, opposed to a monocular reader who does not require any more concentration beyond interpreting words.

Although the lowest scores occurred on monocular readers while higher scores belong to the stereo group, additional research is needed to confirm whether stereo perception may draw upon and enhanced visual memory modality that otherwise would not be involved during monocular reading.

\subsection{Venues for future research}

During this study, the short amount of time between reading the story and answering the questionnaire is only amenable to a study for short term memory. A longer term study would allow for a more personalized intervention in which all comfort parameters can be maximized for particular subjects and long term memory may be tested.

Additional work is needed to discover a better way to present the stereo text. It is very important to fine tune the algorithm so that it produces higher quality text; the most prevalent complaint from participants was the difficulty of making out the characters. The objective of improving the text is so that reading stereographically becomes second nature. Since there exists a significant correlation between comfort and performance, we can expect that future experiments that augment comfort levels will increase memory performance.

Stereoscopic reading may provide a new aim for the exploration of eye strain during prolonged reading. Normally a reader gets tired of reading from one or both of the following conditions: the muscles that control vergence become strained after sustaining eye convergence at same angle for extended periods of time, and/or accommodation muscles become tired while holding the shape of the lens for prolonged time. A stereogram provides a way of constantly varying the depth at which an object is seen, therefore allowing different vergence angles. In terms of continuous focus, changing the eyes' lens focal point is achieved simply by changing the distance of the projection plane. Merely increasing the distance from the eyes to the screen changes eye focus. Recent research focused on new algorithms to reduce eye strain while viewing stereoscopic-pair images [McVeigh et al. 1996], therefore similar techniques can be applied to improve on stereo reading.

We believe testing at different depths may trigger distinct responses, firstly, different disparity brain cells may trigger [Portas 2000]; secondly, dramatically protruding text may be more interesting to subjects, hence affecting their experience. 


\section{References}

[Crawford et. al.] H.J. Crawford, B. Wallace, K. Nomura, and H. Slater, 1986, "Eideticlike imagery in hypnosis: Rare but there". American Journal of Psychology, 99, pp. 527-546

[Cumming \& DeAngelis 2001] Cumming, BG and DeAngelis, GC (2001), The physiology of stereopsis. Annu Rev Neurosci.24:203-38

[Eichenbaum 1997] Eichenbaum, H. 1997 Declarative memory: insight from cognitive neurobiology. A. Rev. Psychol. 48, pp. 447-572

[Emoto et al. 2005] M. Emoto, T. Niida, and F. Okano, "Repeated vergence adaptation causes the decline of visual functions in watching stereoscopic television," J. Disp. Tech. 1, 328-340 (2005)

[González 2000] Archivos de la Sociedad Española de Oftalmología "Los Estereogramas de puntos al azar, la oftalmología y Ramon y Cajal" http://www.oftalmo.com/seo/2000/12dic00/14.htm accessed Oct 6,2007

[Julesz 1972] B. Julesz, 1972, "Cyclopean perception and Neurophysiology". Invest. Ophthalmol. Vis. Sci. 11 (6) 540

[Magnussen 2001] Magnussen, S. 2001, The psychology of visual memory. The International Encyclopedia of the Behavioural and Social Sciences,Volume 24, pp. 1626416266

[McVeigh et al. 1996] J.S. McVeigh, M.W. Siegel, and A.G. Jordan. Algorithm for automated eye strain reduction in real stereoscopic images and sequences. Proc. SPIE Vol. 2657: Human Vision and Electronic Imaging pp. 307 - 316, 1996.

[Morland 1976] D. Verne Morland, 1976, "Computer-Generated Stereograms A New Dimension for the Graphic Arts" Proc.ACM SIGGRAPH 7610 19-24

[Nakagawa 2005] K. Nakagawa, S.Nomura, T. Nagashima, "Estimation of the Exact Moment of Human Cognition of RDS", 2005 Asian conference on sensors and the international conference on new techniques in pharmaceutical and biomedical research proceedings, 57 pp. $70-73$

[N.Quian 1997] N.Quian, 1997, "Binocular Disparity and Perception of Depth" Neuron, Vol 18, pp,359-368

[Petz 2003] C. Petz, B. Goldlücke, M. Magnor 2005 "Hardware-accelerated Autostereogram Rendering for Interactive 3-D Visualization" Proc. SPIE Electronic Imaging EI'03 5006 pp. 359-366 
[Portas 2000] C.M Portas, B.A Strange, K.J. Friston, R.J.Dolan, C.D. Frith, 2000, "How does the brain sustain a visual percept?" Proceedings of the Royal Society B: Biological Sciences 267 pp. 854-850

[Schroeder et al. 1998] W.Schroeder, K. Martin, B. Lorensen, The Visualization Toolkit, An Object-Oriented Approach to 3D Graphics, 2nd Edition Prentice Hall 1998

[Swain 1997] C.Swain, 1997, "Integration of Monocular Cues to Create Depth Effect", ICASSP'97 Volume4 p. 2745

[Thimbleby et al. 1994] Thimbleby, H.w. Inglis, S. Witten, I.H. 1994 Displaying 3-D images: algorithms for single-image random-dot stereograms. Computer 27 pp. 38-48

[Wheatstone 1838] C. Wheatstone 1838, "Contributions to the Physiology of Vision. Part the First. On Some Remarkable, and Hitherto Unobserved, Phenomena of Binocular Vision" Philosophical Transactions of the Royal Society of London, Vol.128, pp. 371-394 


\section{A Stereo disparity as a tool to ascertain similarities or differences}

Using stereo disparity, it is possible to identify differences among similarities between objects; conversely it is also possible to identify similarities among different objects. To put the idea of "differences among similarities" in context, let us look at Figure 15. In this figure we can see two very similar photographs of the corner of an office. Both pictures were taken from a fixed position, therefore there is no change in vantage point between the two images. There are five differences between these two pictures, some being more apparent than others.
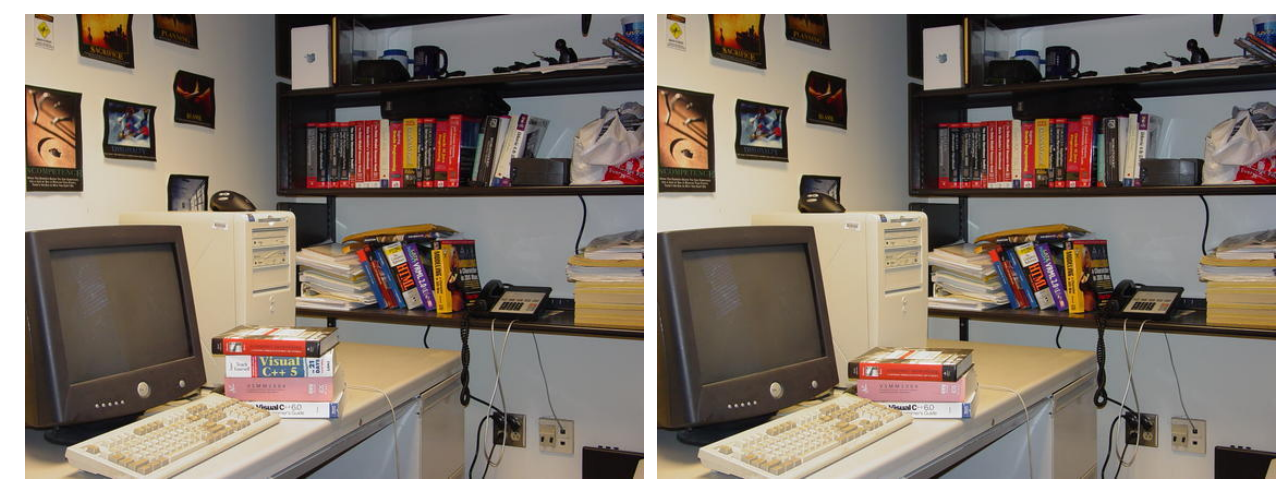

Figure 15: Noticing differences among similarities

By looking at the photographs alternatively, a viewer interested in identifying these five differences could be staring at the images for a long time and still have difficulty discovering them. The differences may be so subtle that glancing repetitively over the same area will not easily hint a change. In a sense, the differences would remain camouflaged in plain view. Perhaps the easiest differences to discover would be the missing book from the stack on the desk, as well as the mouse displacement on top of the computer; while the most difficult differences to notice are: book displacements on second shelf, the dangling power cable between first and second shelves, and the slight twist on the auricular cord on telephone.

Stereo disparity allows us to discover the differences between both pictures immediately and effortlessly ${ }^{10}$. Viewing both images dichoptically, we can instantly notice all differences at once. The locations of the discrepancies will become apparent immediately and are noticeable due to a scintillation effect that results from unmatched disparity between the two retinas.

\footnotetext{
${ }^{10}$ Untrained eyes that cannot converge dichoptically on the images may require the aid of a stereoscope or a haploscope
} 
In the same way we can see differences as those illustrated in figure 15, it is also possible to consolidate similarities from apparently dissimilar objects. Figure 16 illustrates an example of a case in which looking at a pair of text columns dichoptically will reveal the letters of a hidden message. Relevant letters of the hidden message become clear when both images are superposed when left eye looks at left asterisk and right eye looks at right asterisk.

\begin{tabular}{|c|c|}
\hline$*$ & $*$ \\
nHulywfrifh & aHahdndiinx \\
jfdatghdbcv & bcd4378dmbn \\
wrioeqwnqyp & cmzrezdnfsj \\
mx2rytjmext & ornugtpwext \\
\hline
\end{tabular}

When viewed adequately the consecutive highlighted letters reveal the message "Hidden text".

Figure 16: Consolidating similarities among dissimilar objects

Visibility of the hidden text is contingent upon preserving the disparity distance between the two views. When looked at carefully, all the relevant letters on the hidden message are repeated in the same position on both columns. The idea of producing depth perception using only text is well known, in fact there exist Single Image Random Text Stereograms that use disparity to either emphasize characters within the text or to portray hidden objects. In the case of Figure 16, we use characters to "hide" the text itself. Figure 17 gives and example of how to emphasize words on a text stereogram.

\begin{tabular}{|c|c|}
\hline \multirow[b]{2}{*}{$*$} & \\
\hline & \\
\hline I took a break & Today I took \\
\hline reading & from reading \\
\hline I stepped outside to & stepped outside \\
\hline enjoy the & enjoy the \\
\hline is shinning so I & shinning so $\mathrm{I}$ \\
\hline fun making & have fun making \\
\hline noise with a fancy gong & noise with a fancy gong \\
\hline
\end{tabular}

When viewed adequately the words "reading is fun" will standout from the text.

Figure 17: Using a text stereogram to emphasize words 


\section{B Code Listing}

\section{fonts.h}

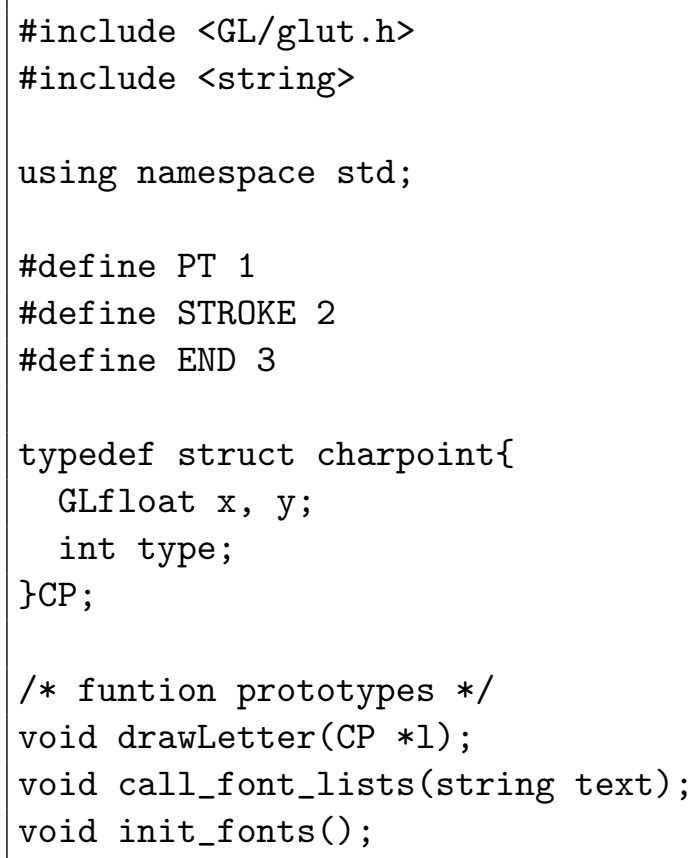

fonts_dlists.cpp

Implements the 3-D alphabet using display lists fonts_dlists.h

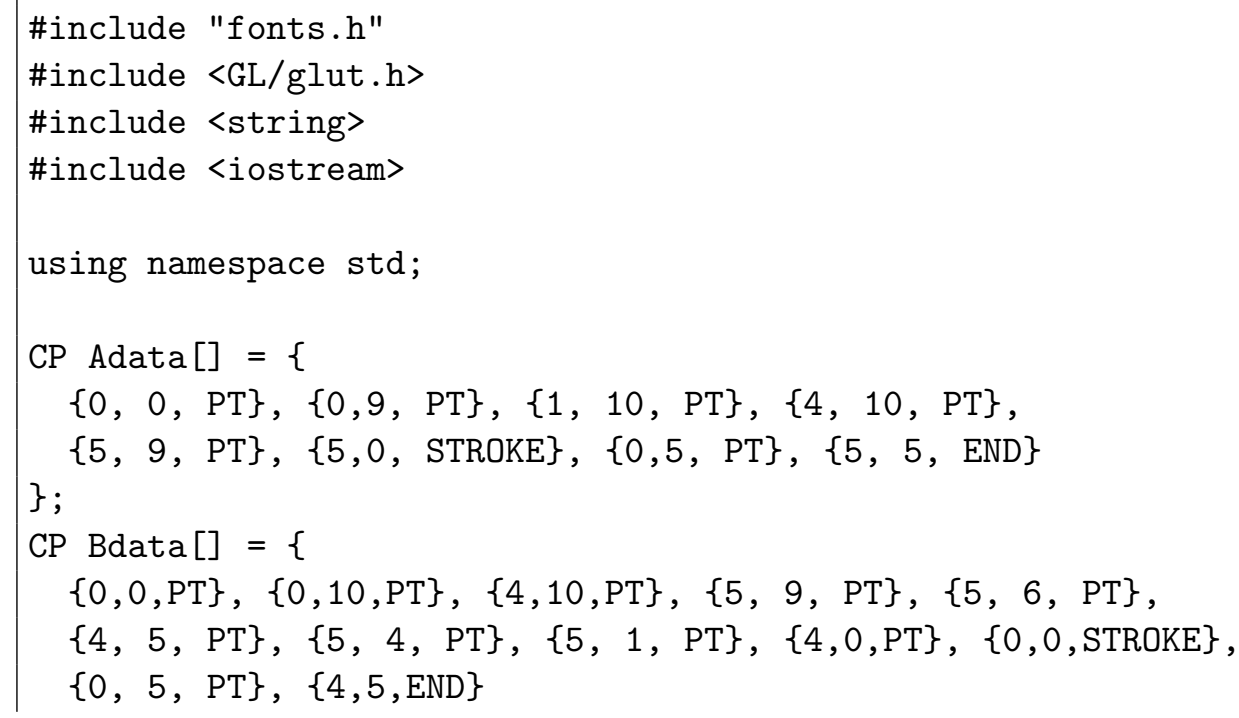




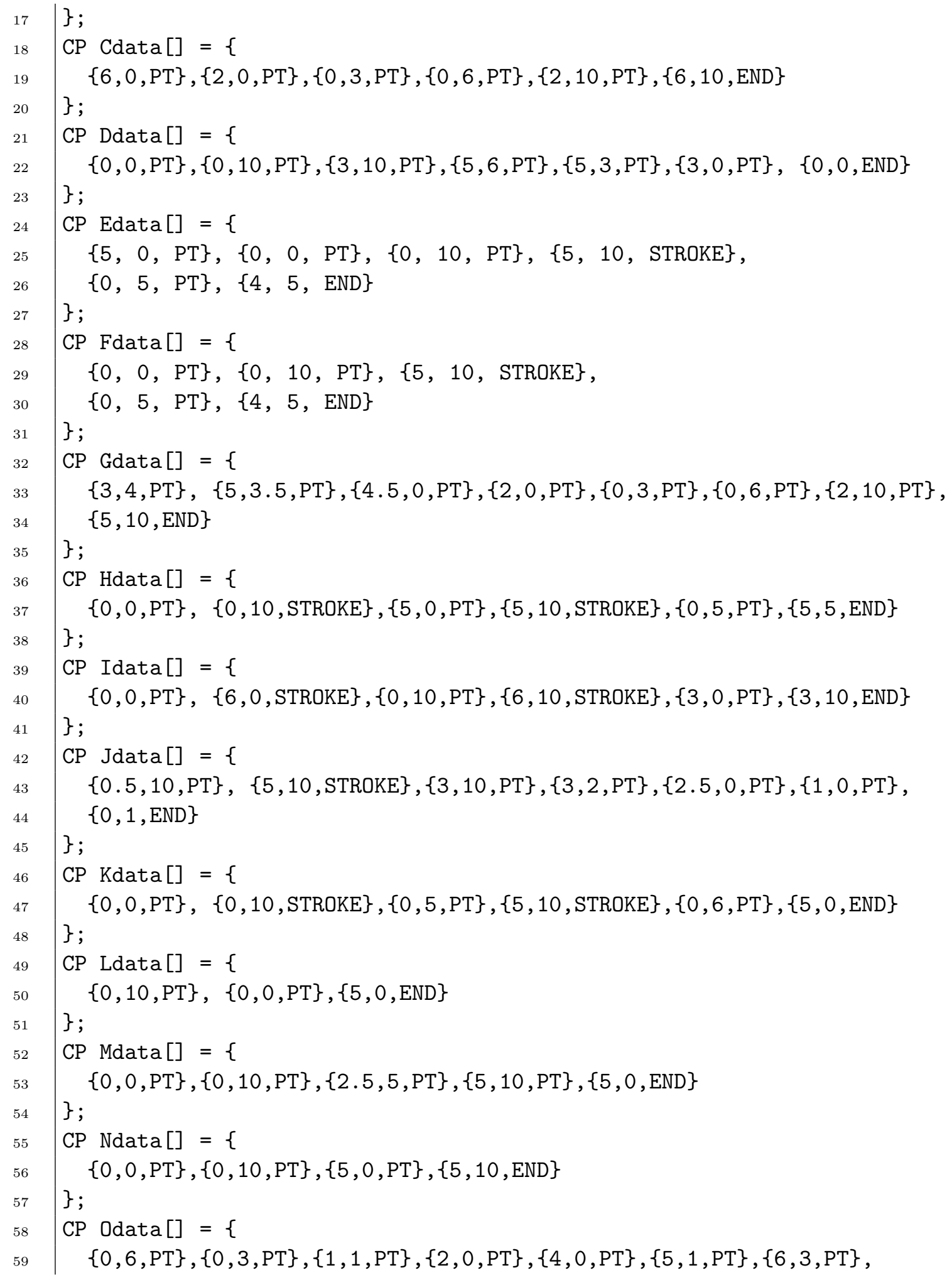




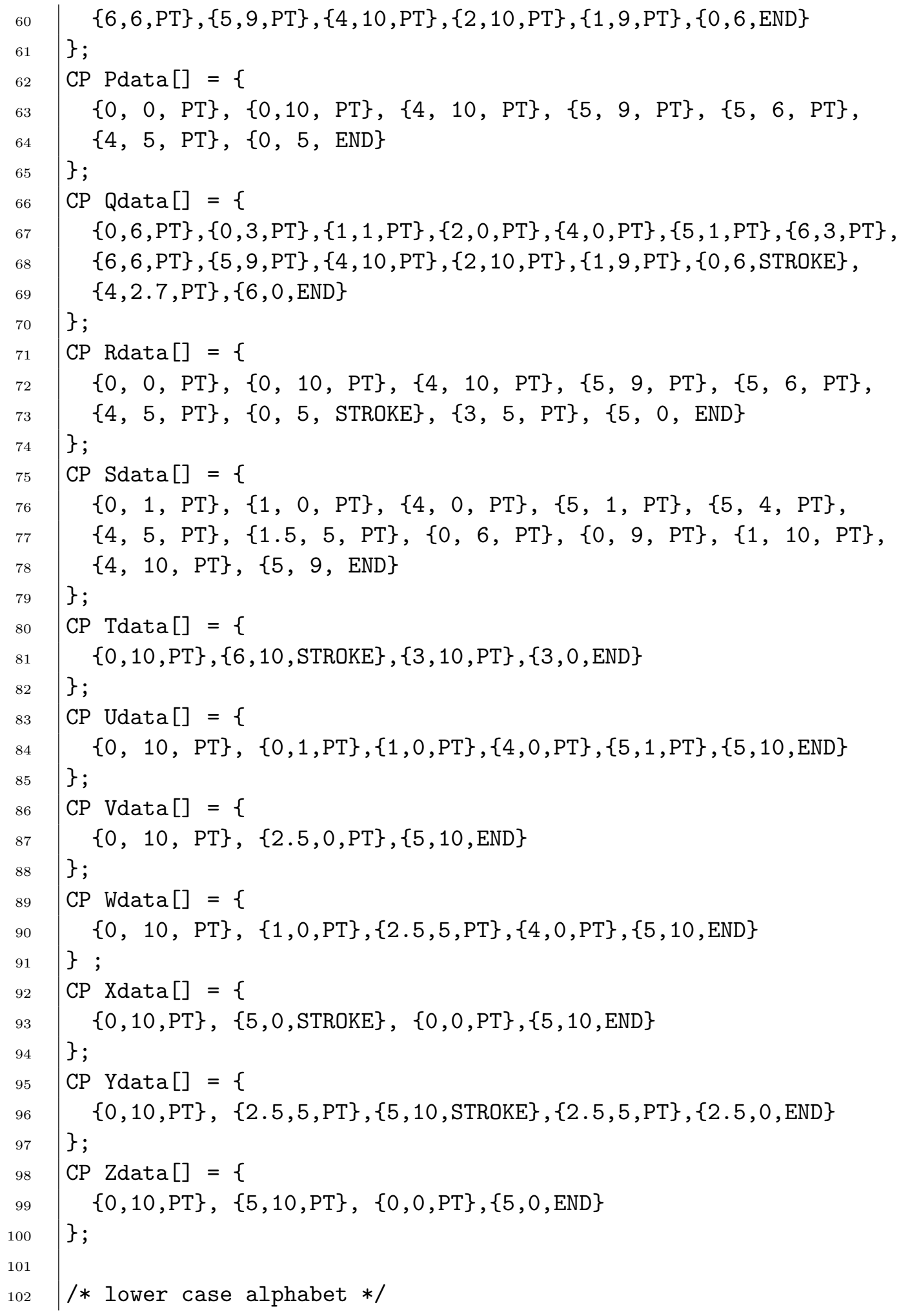




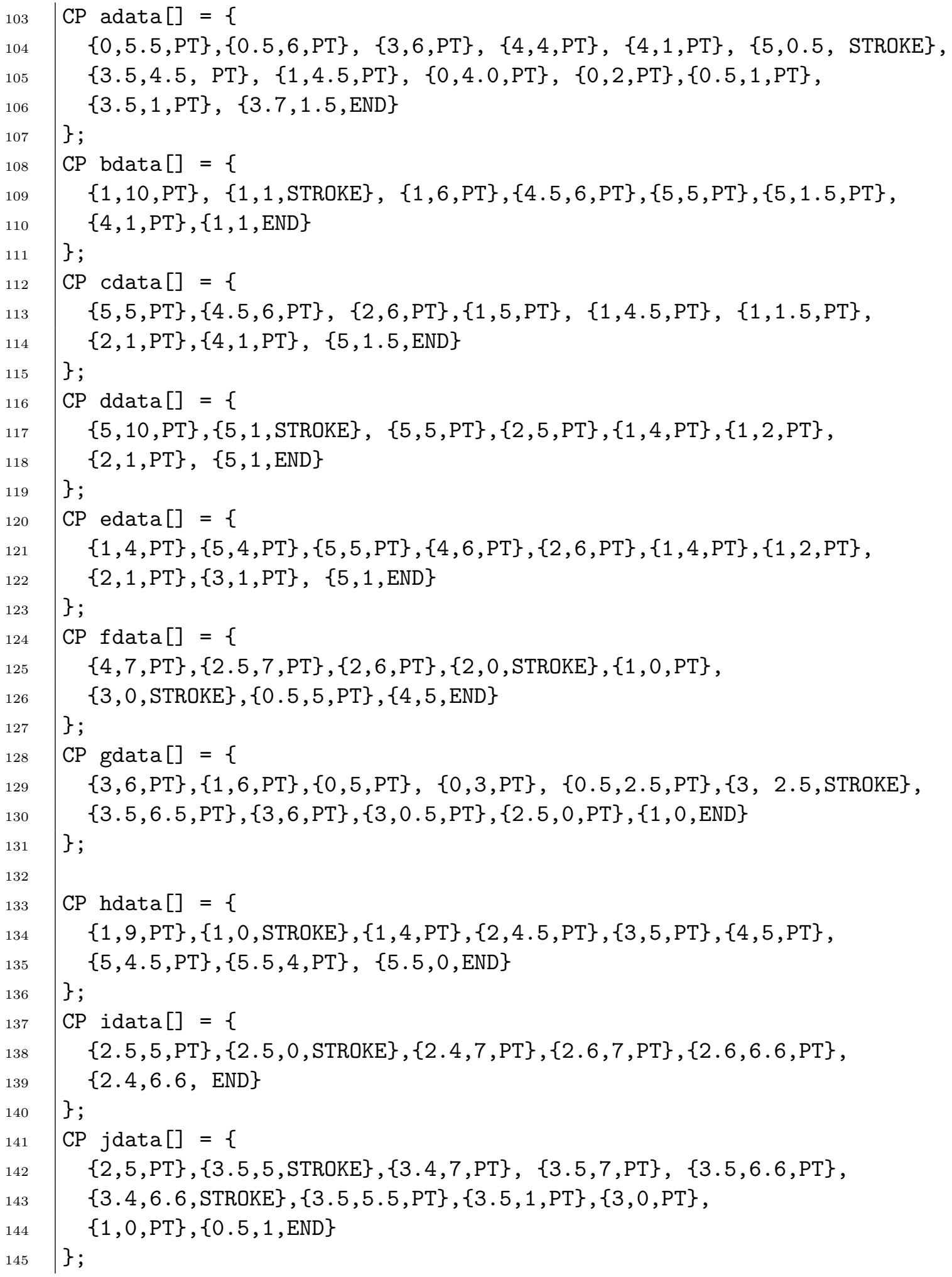




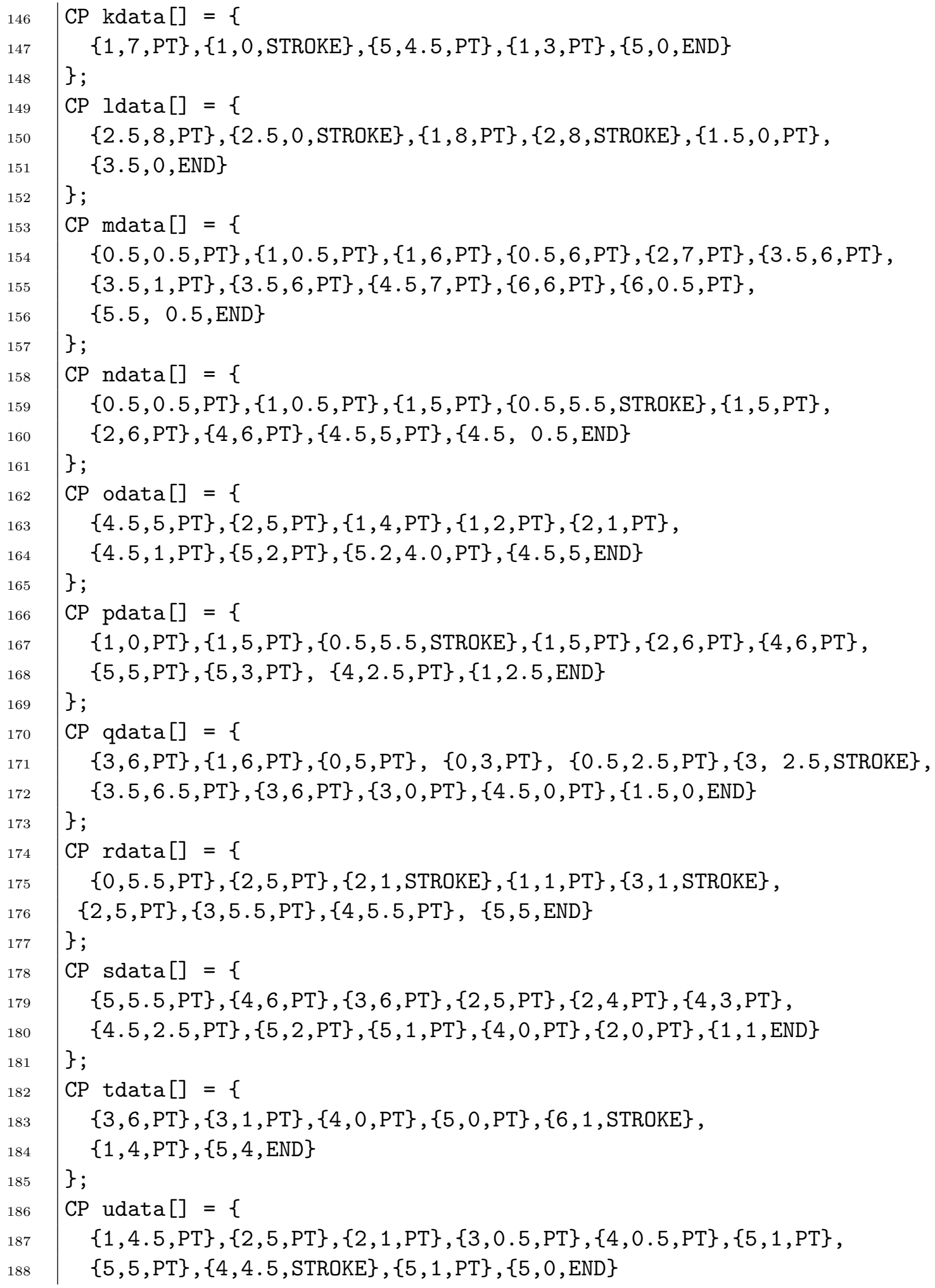




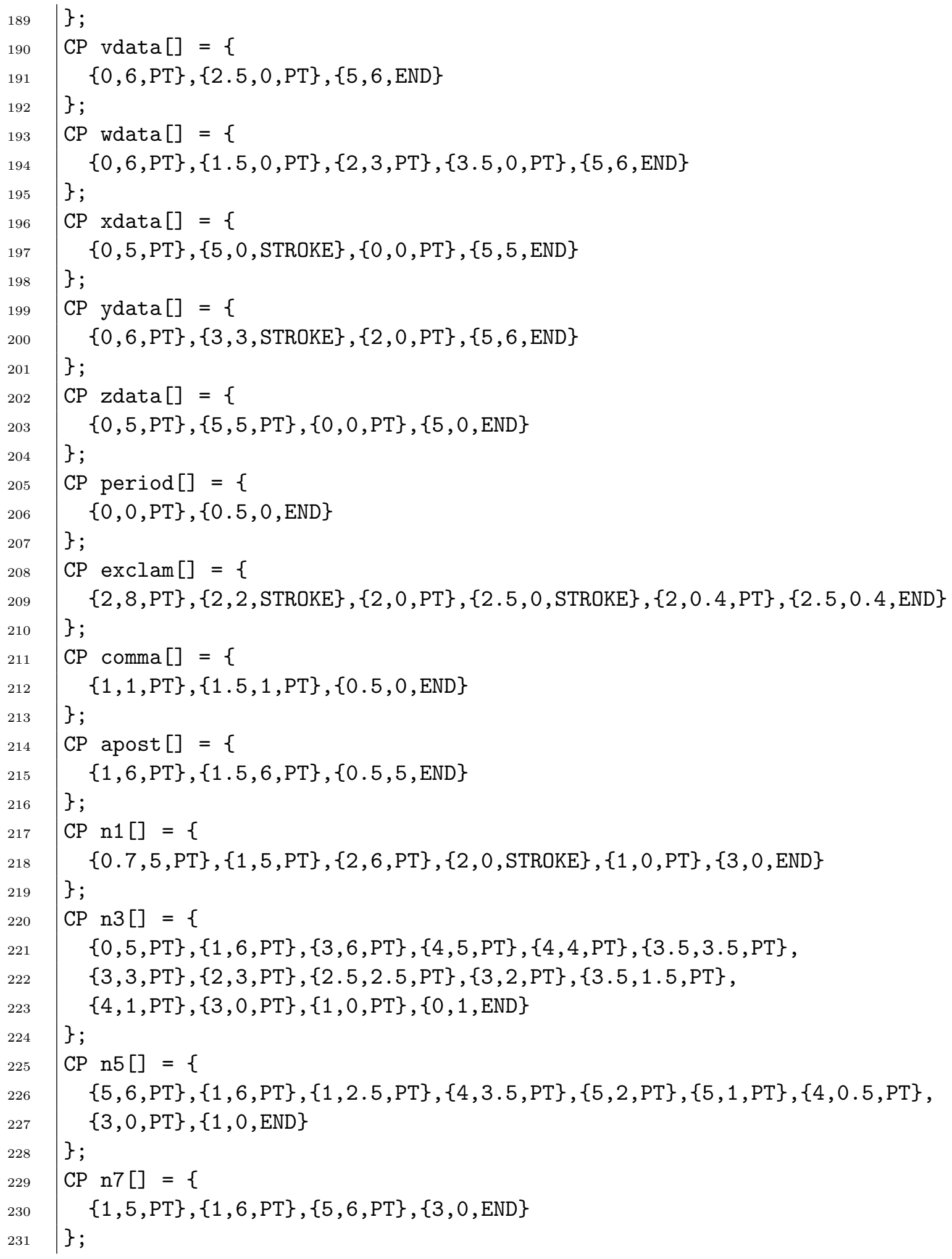




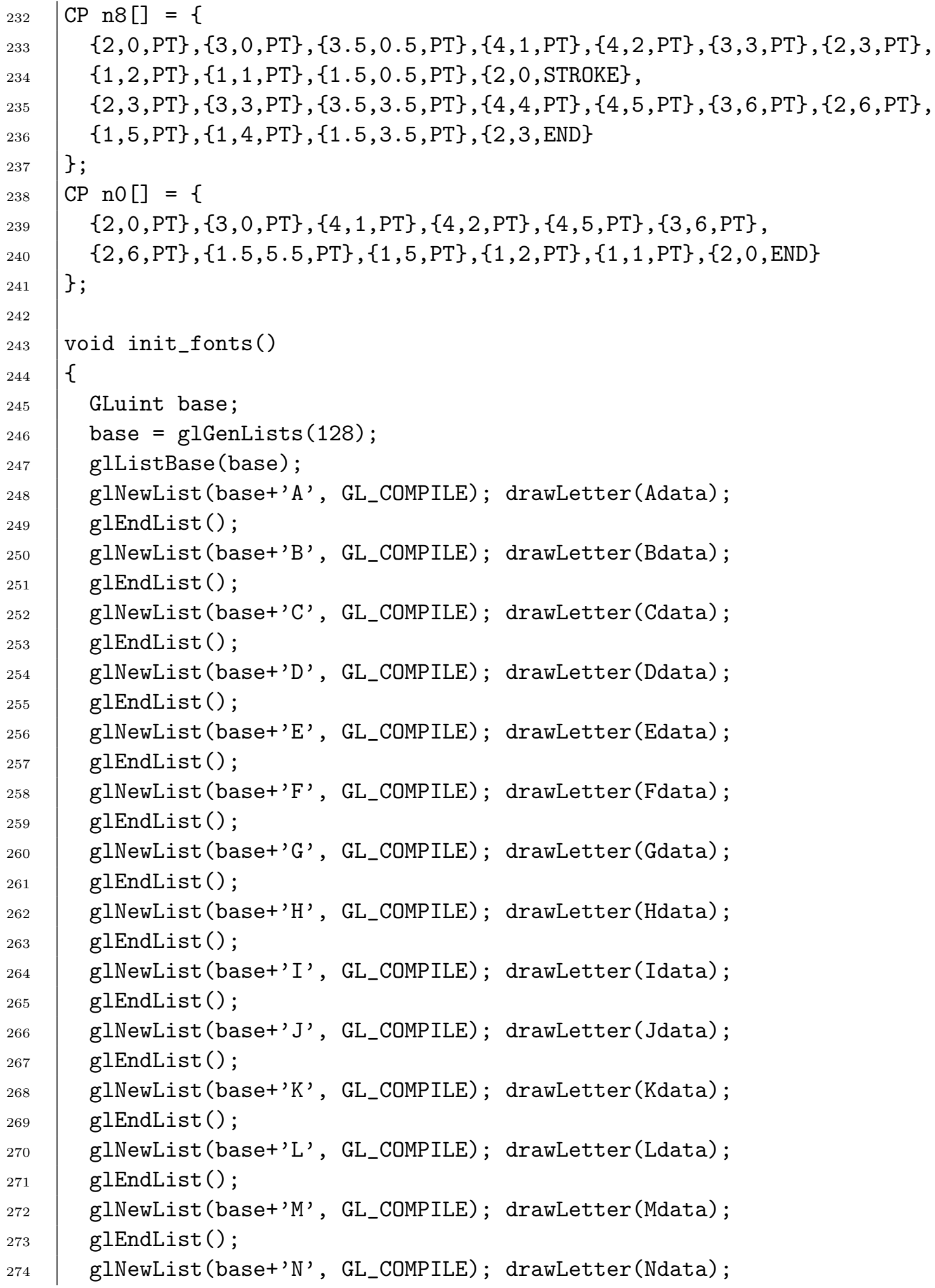




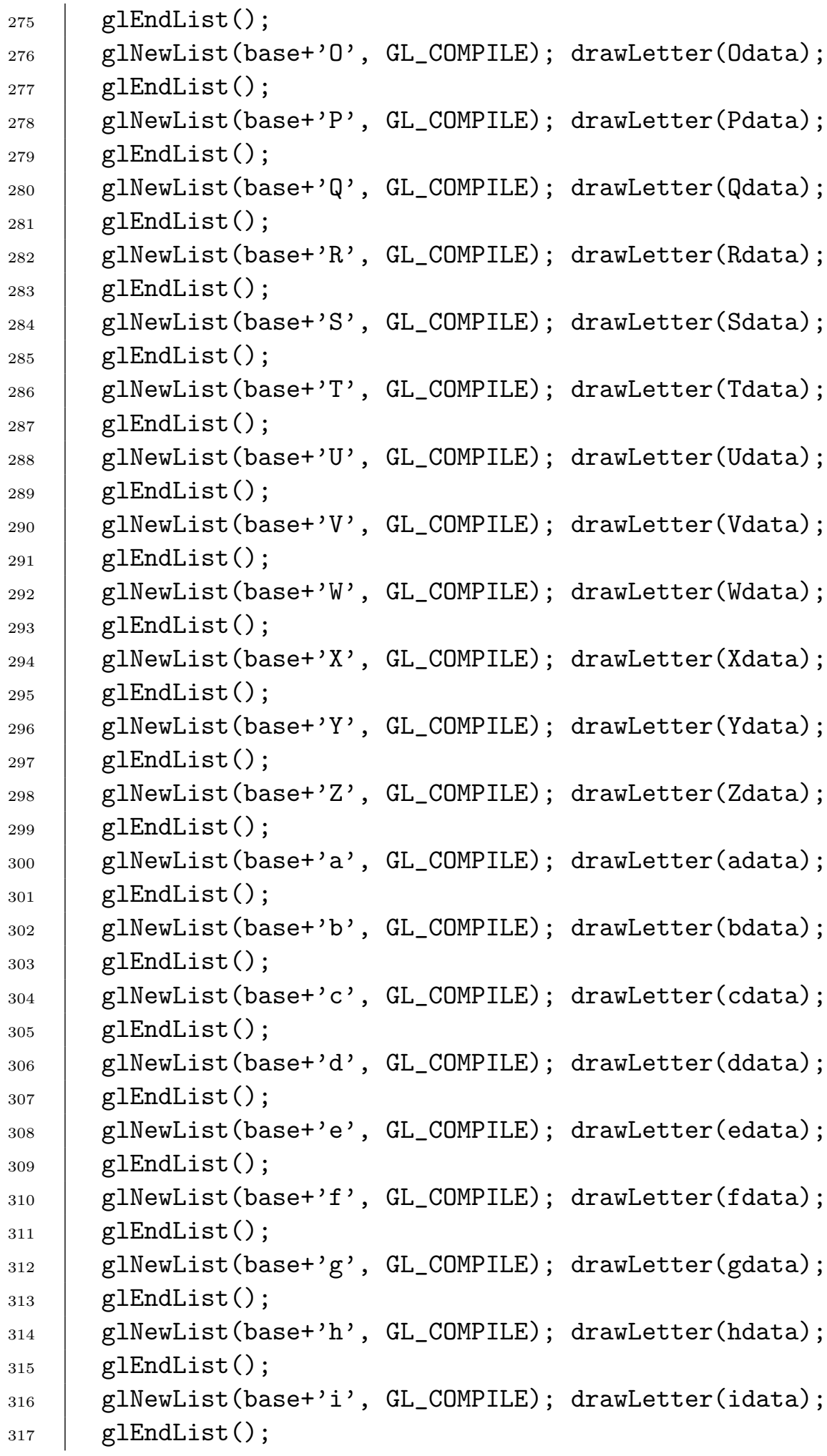




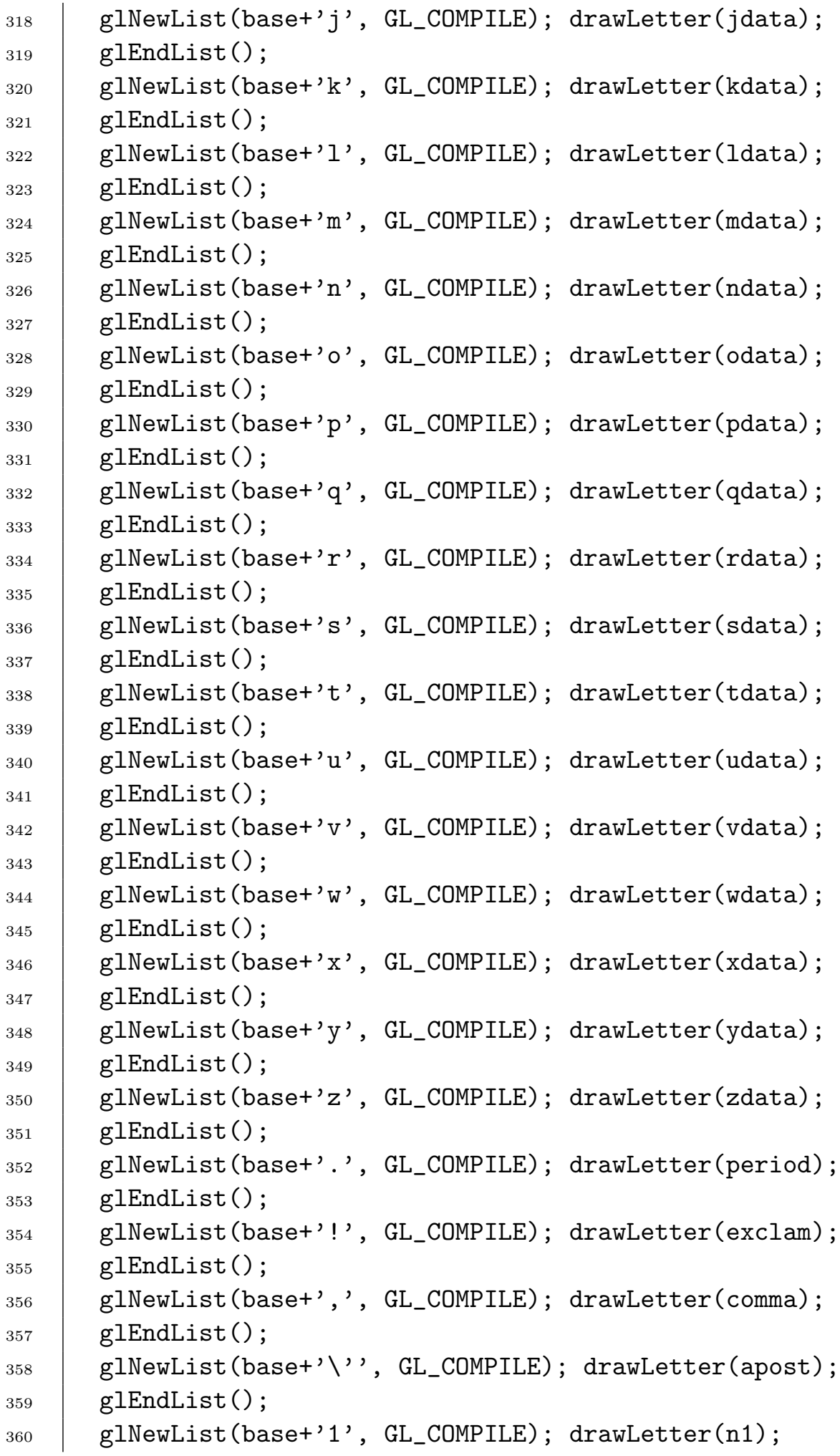




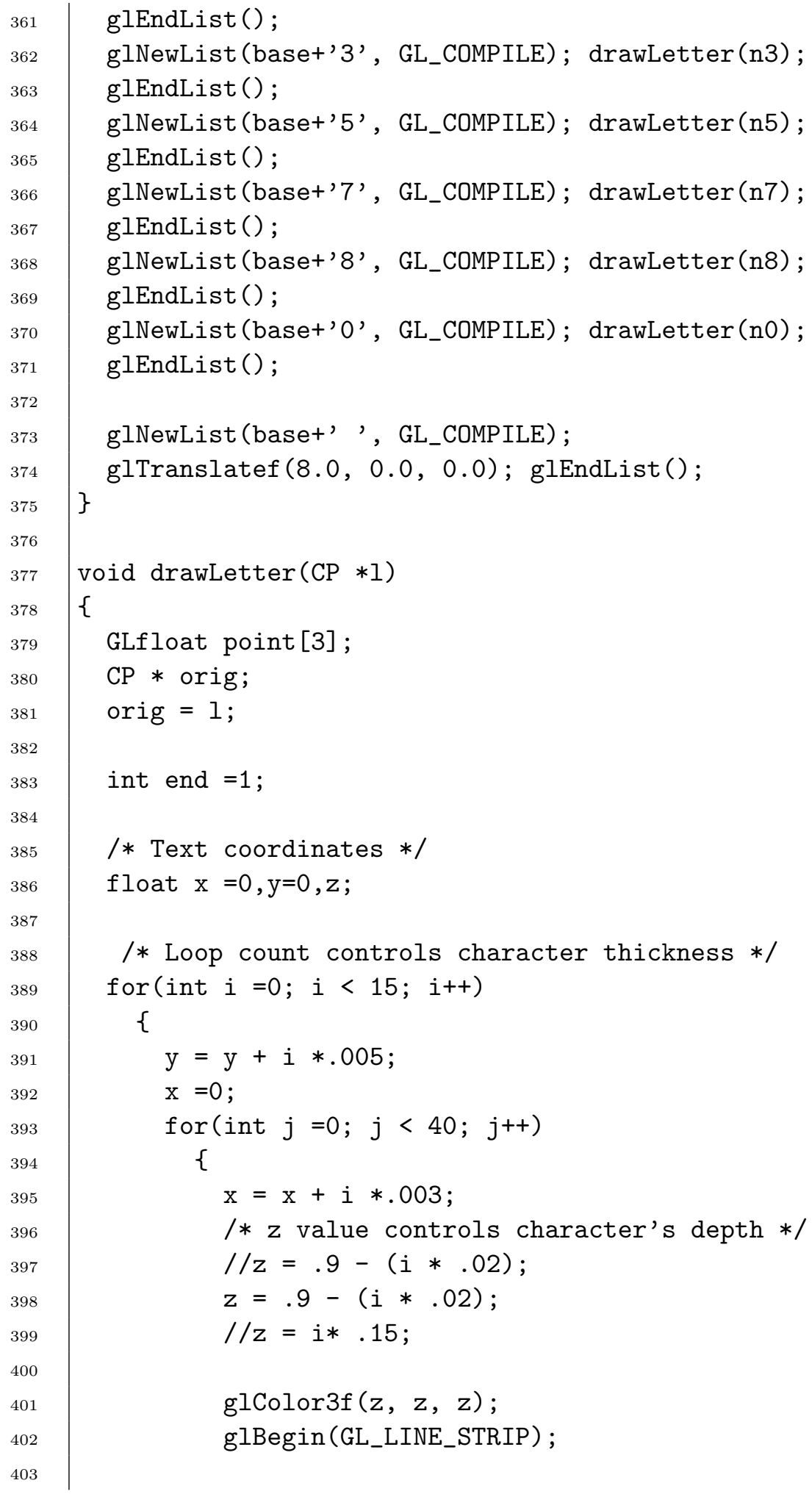




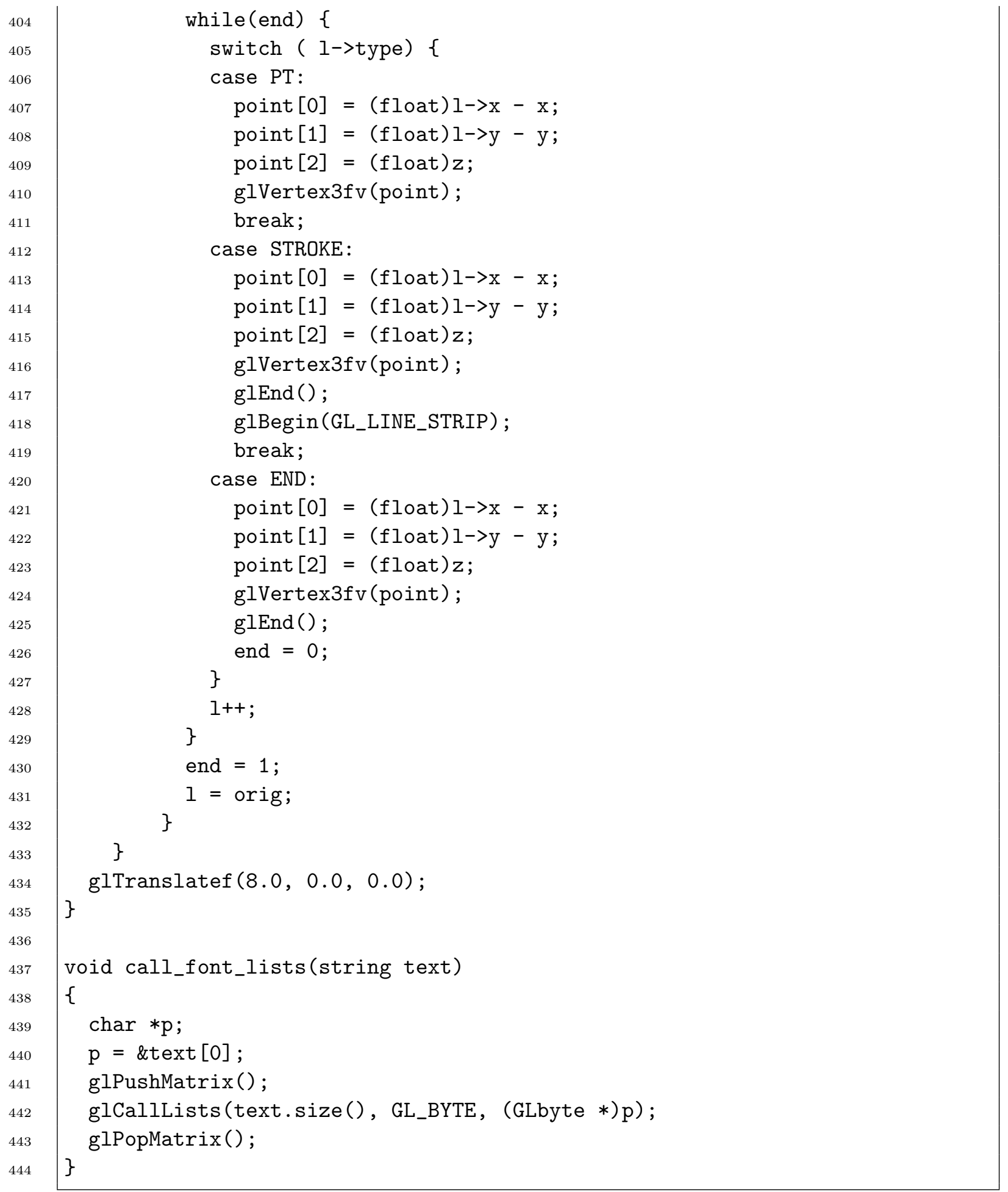




\section{randompattern.h}

Establishes the size of the stereogram and function prototypes used in randompattern.cpp

\#include <GL/glut.h>

\#define pattern_width 1018 //\#define pattern_width 1151

\#define pattern_height 650

//\#define pattern_width 512

//\#define pattern_height 512

void create_Random_pattern(int granularity, int color);

void create_image_pattern();

void modify_random_pattern ();

void draw_modified_pattern(int helper_dots);

unsigned char* ppmRead(char* filename, int* width, int* height); 


\section{randompattern.cpp}

Implements the random pattern, reads in the background images and computes stereogram pixels.

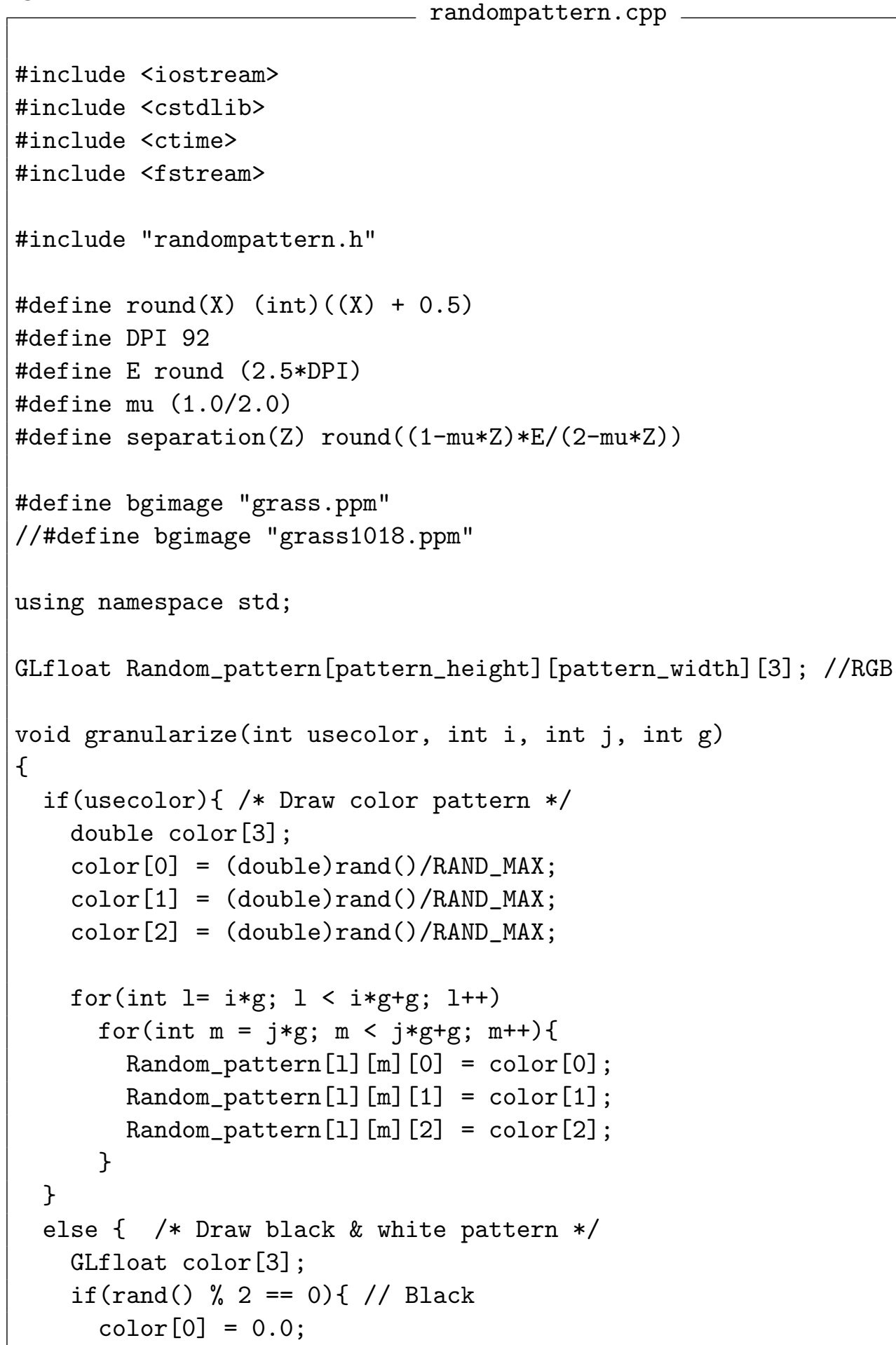




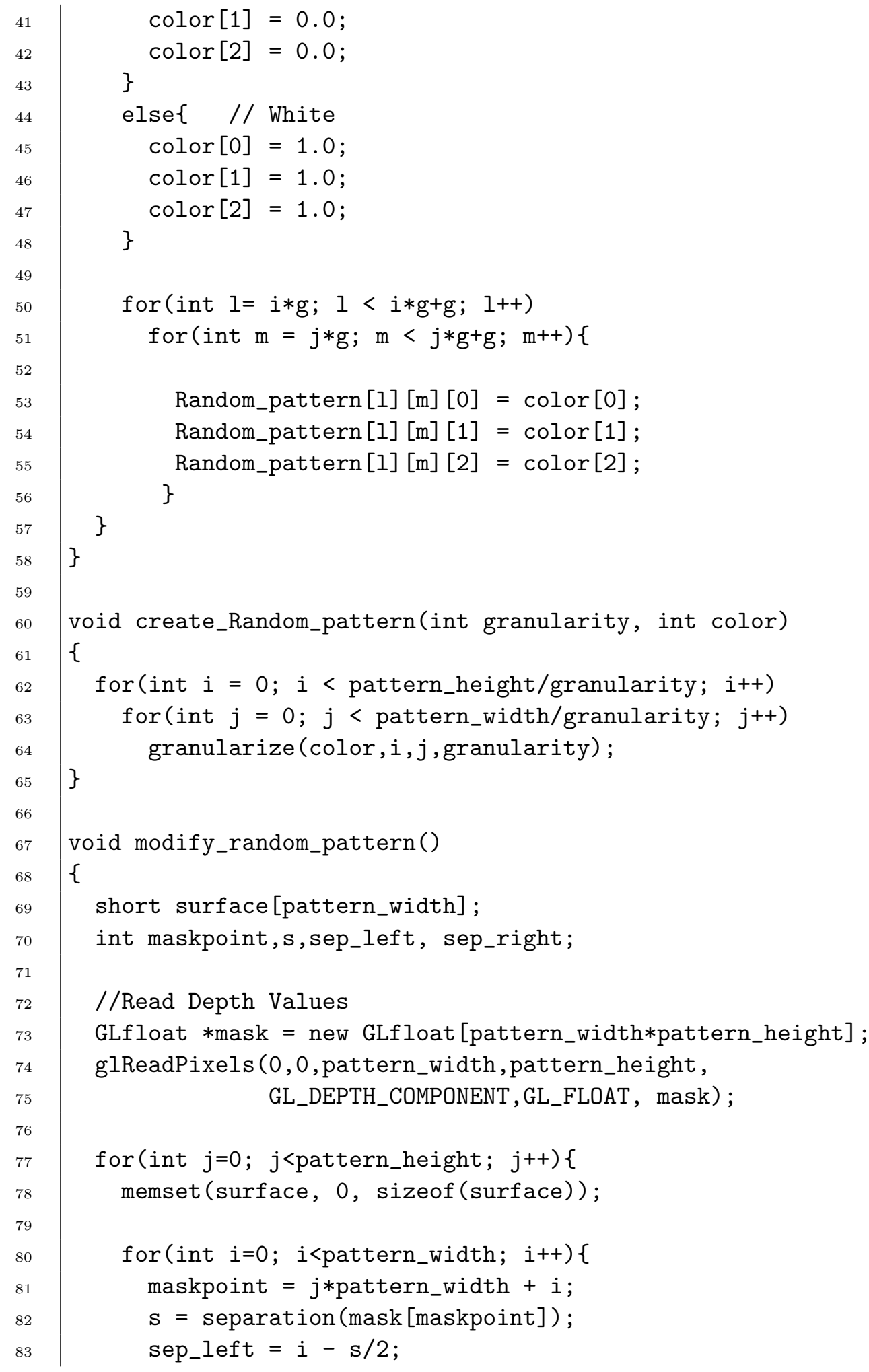




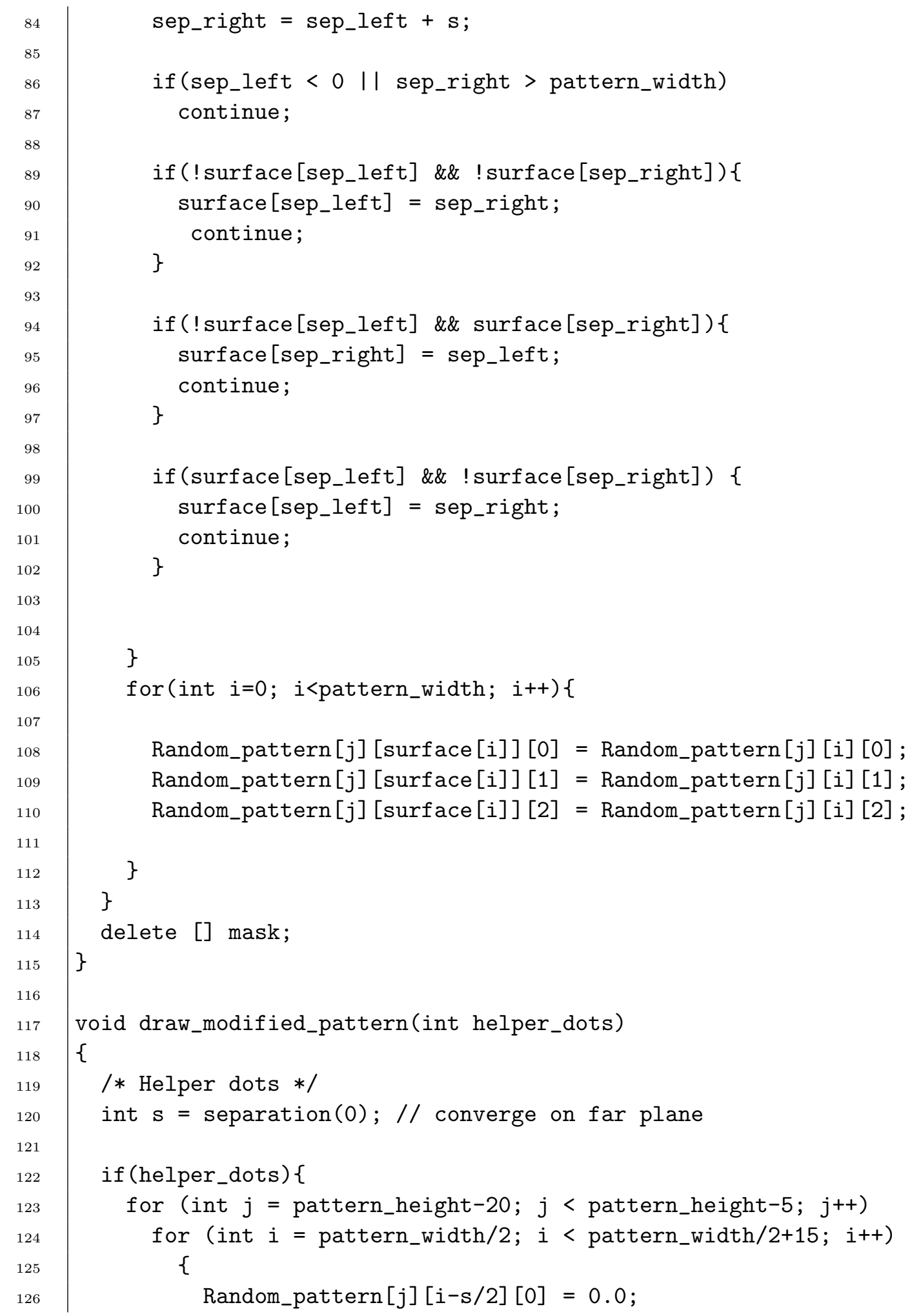




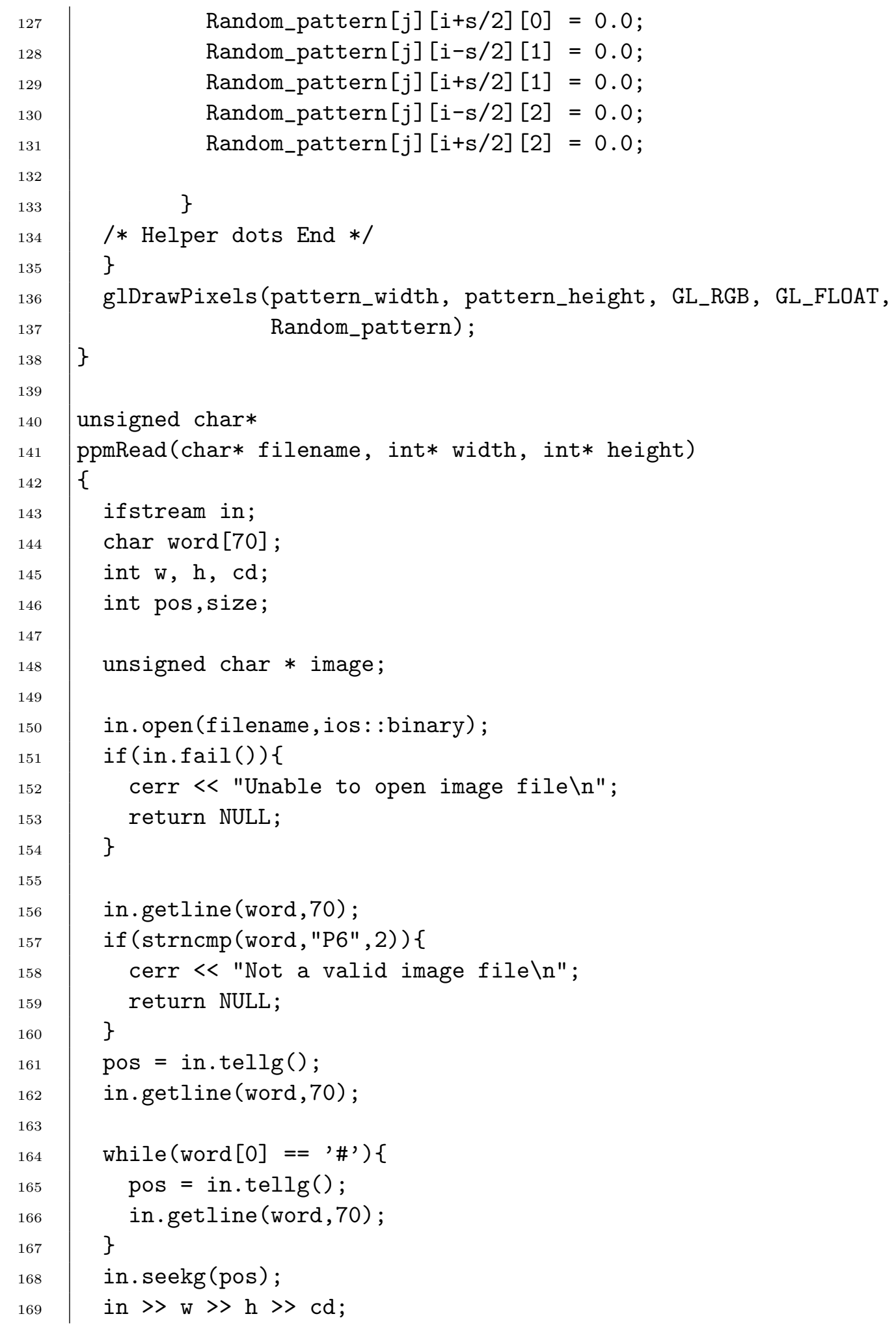




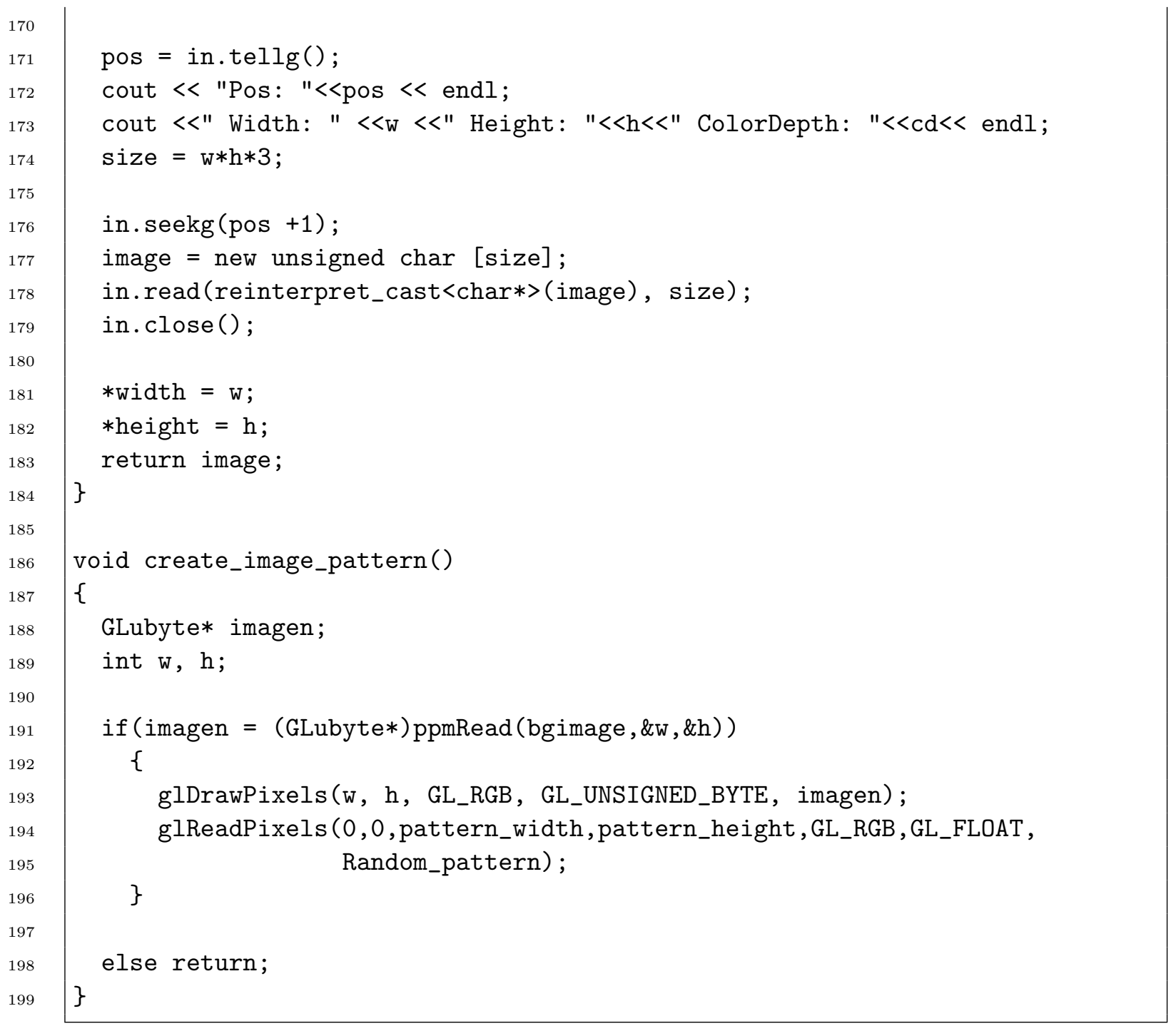


stereogram.cpp Implements the Graphical User Interface for the autostereogram window.

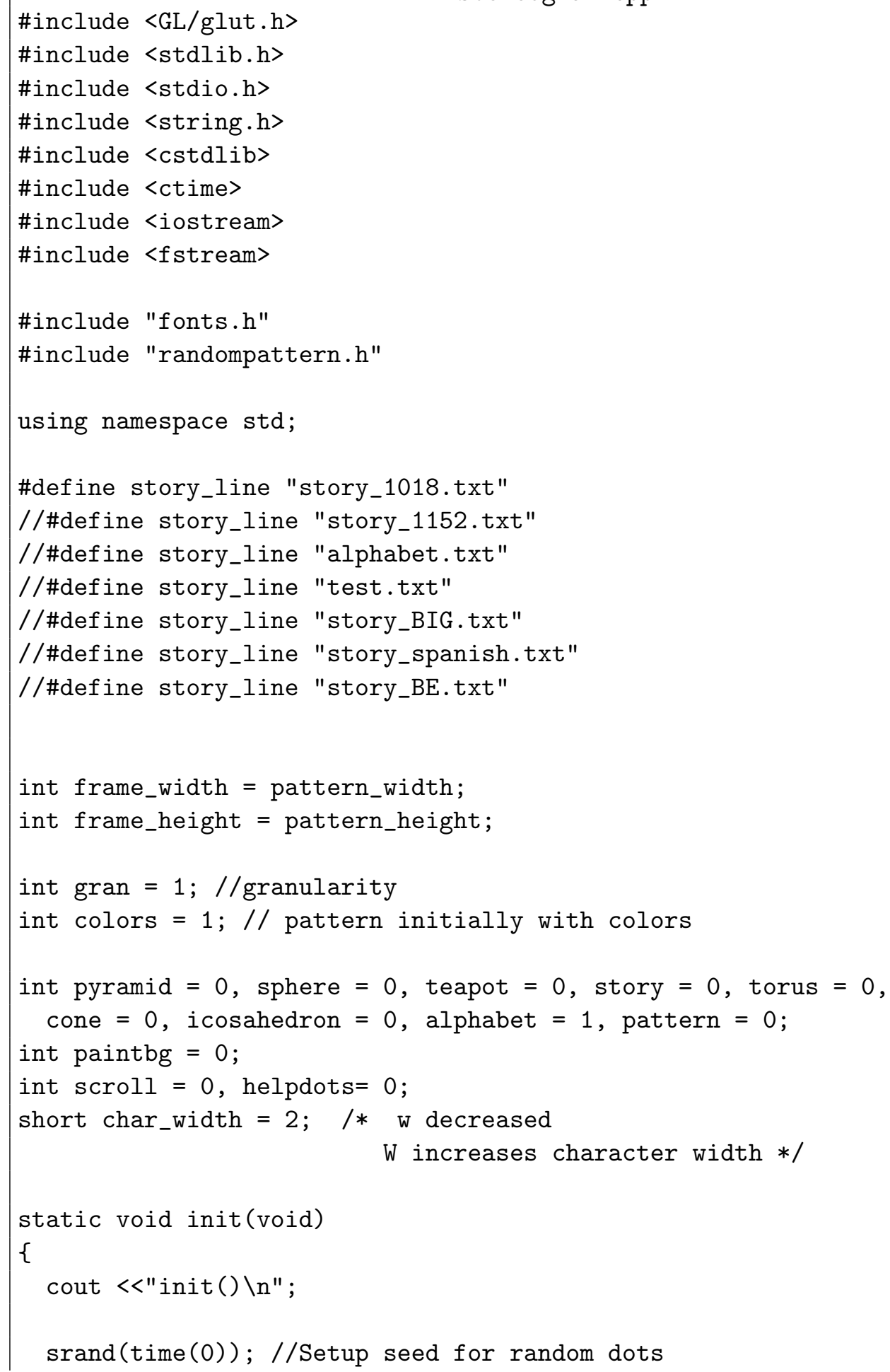




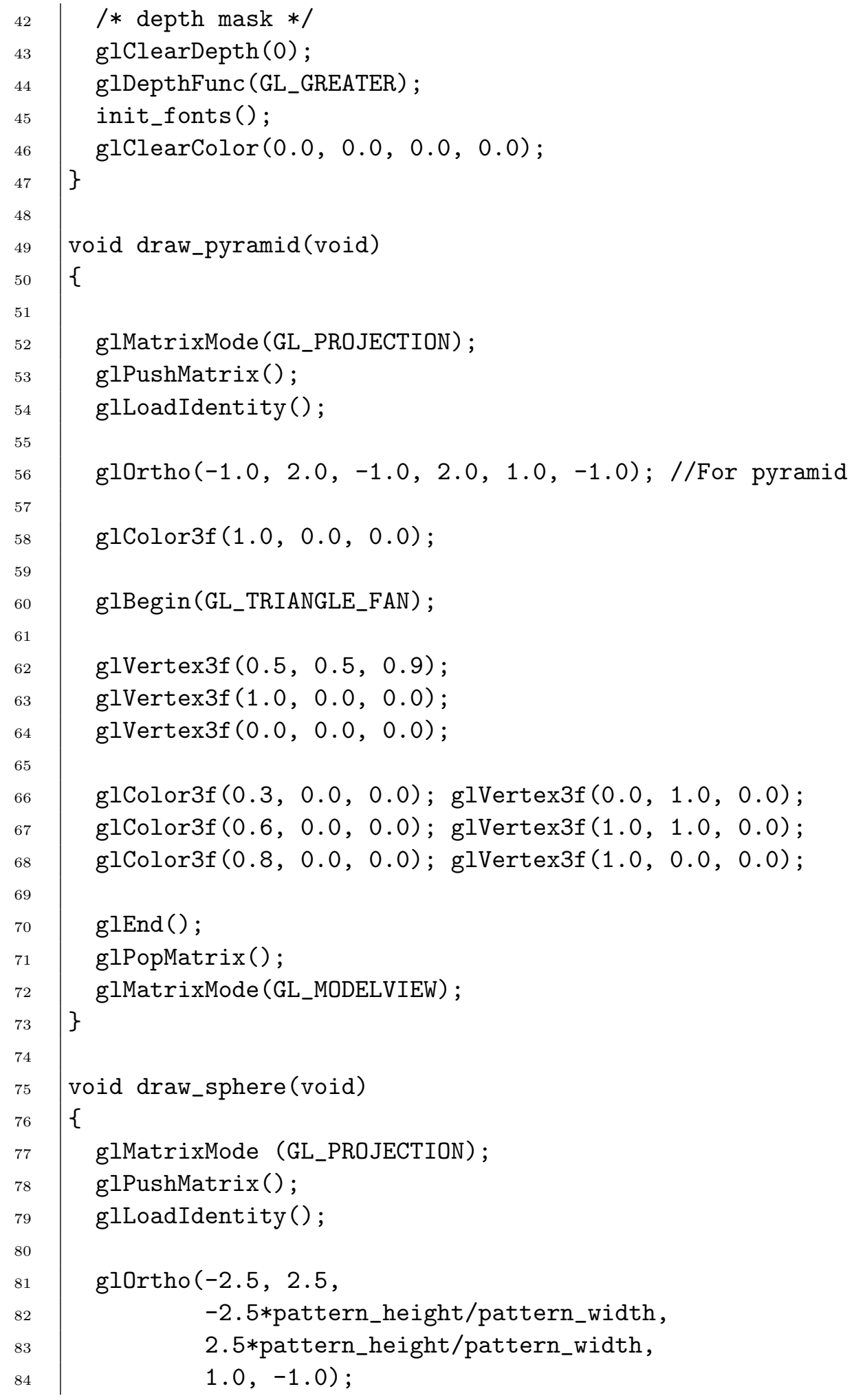




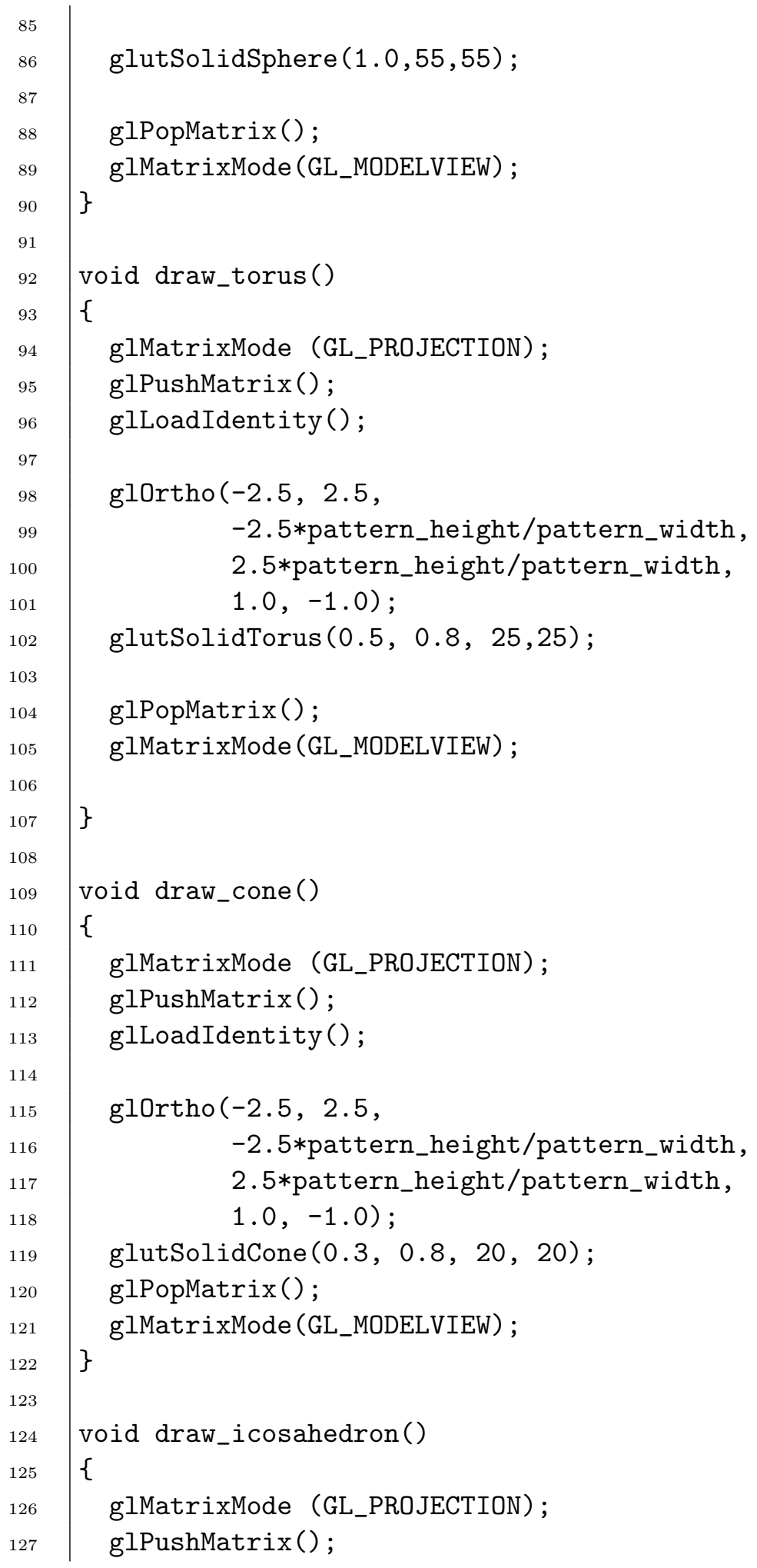




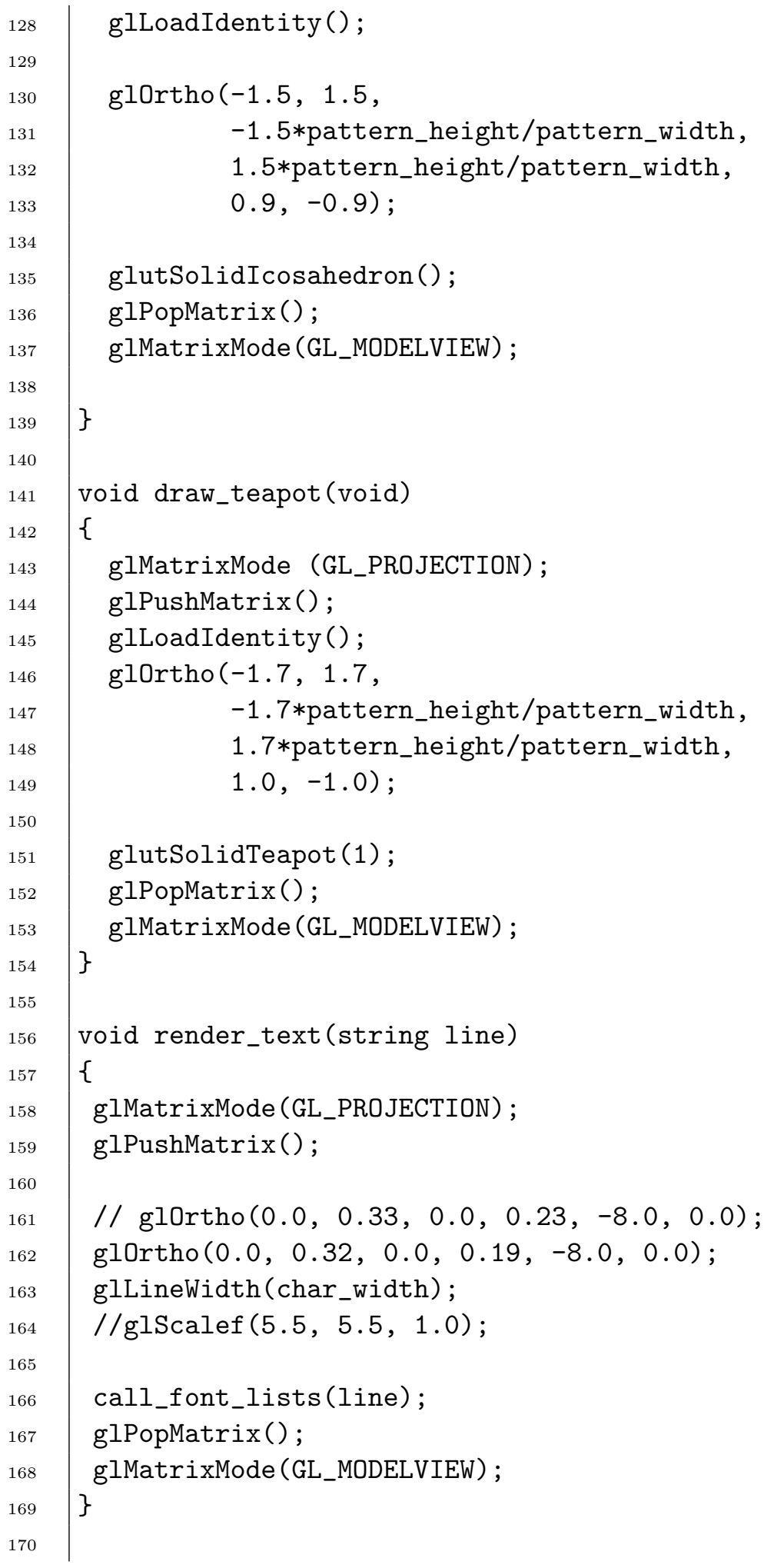




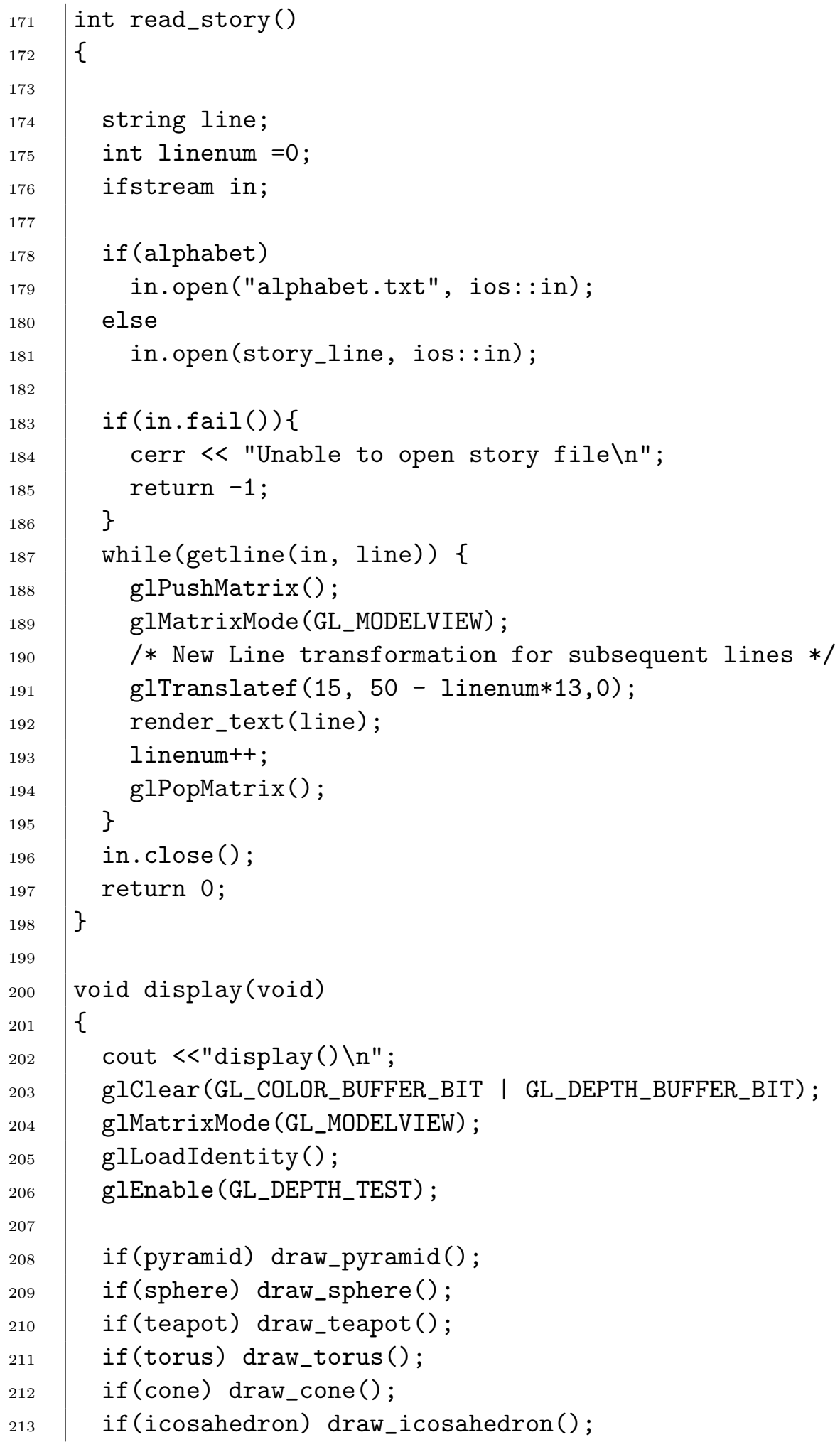




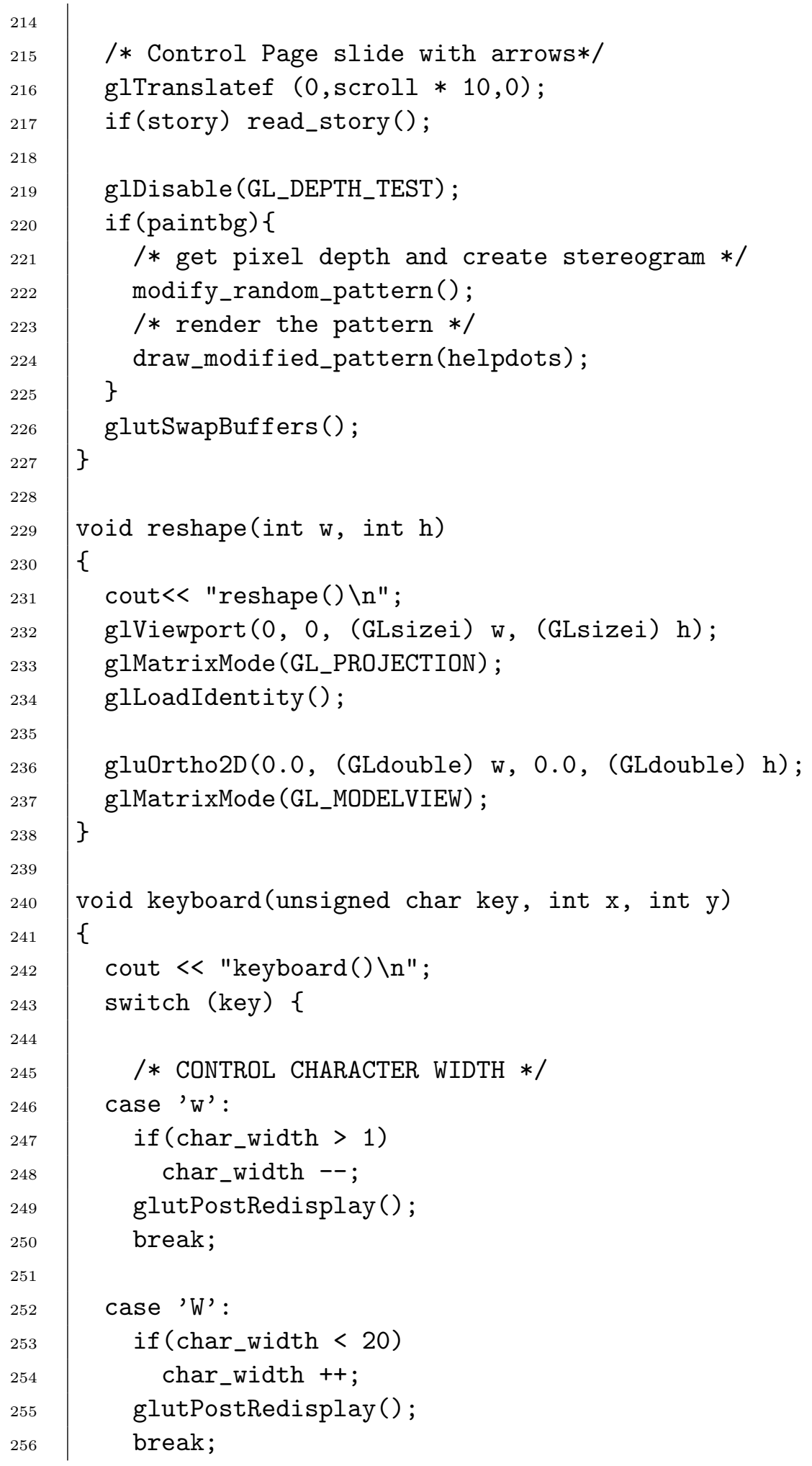




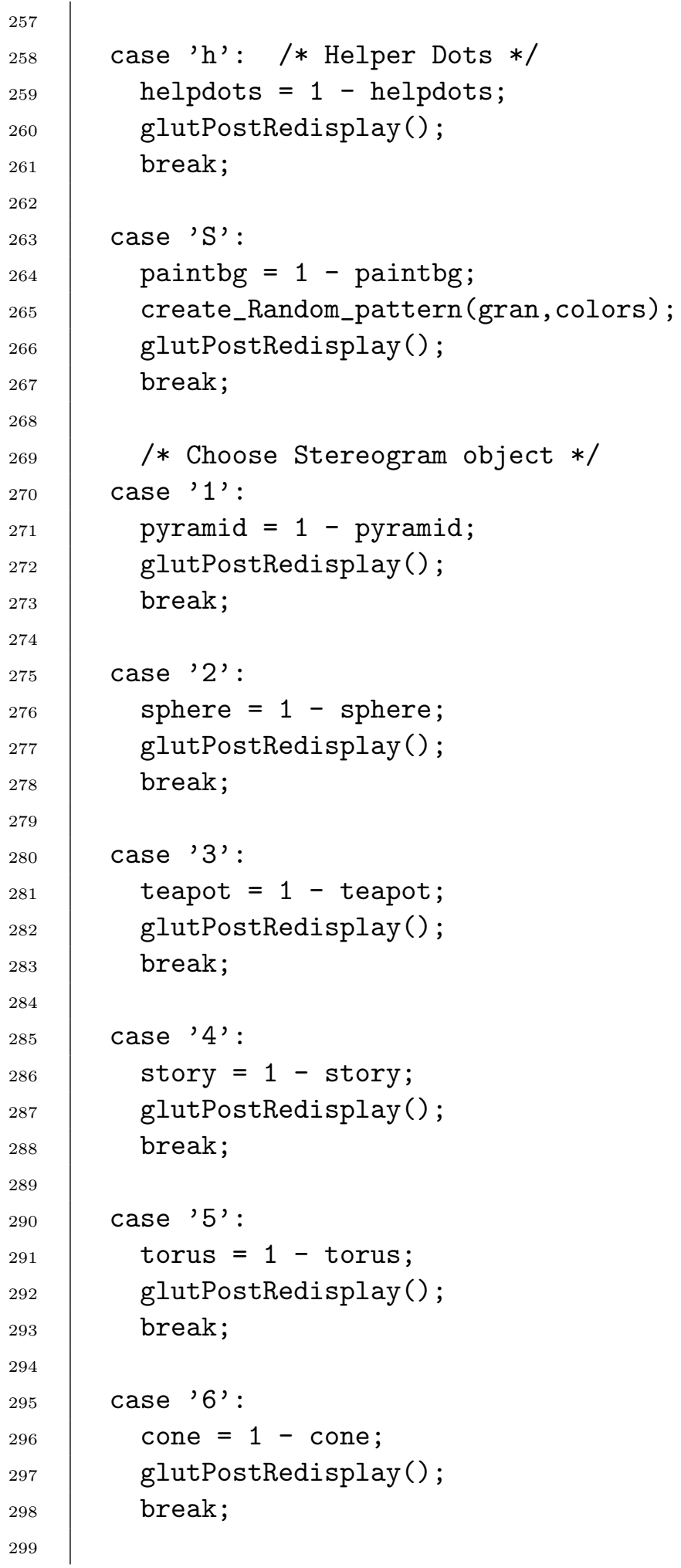




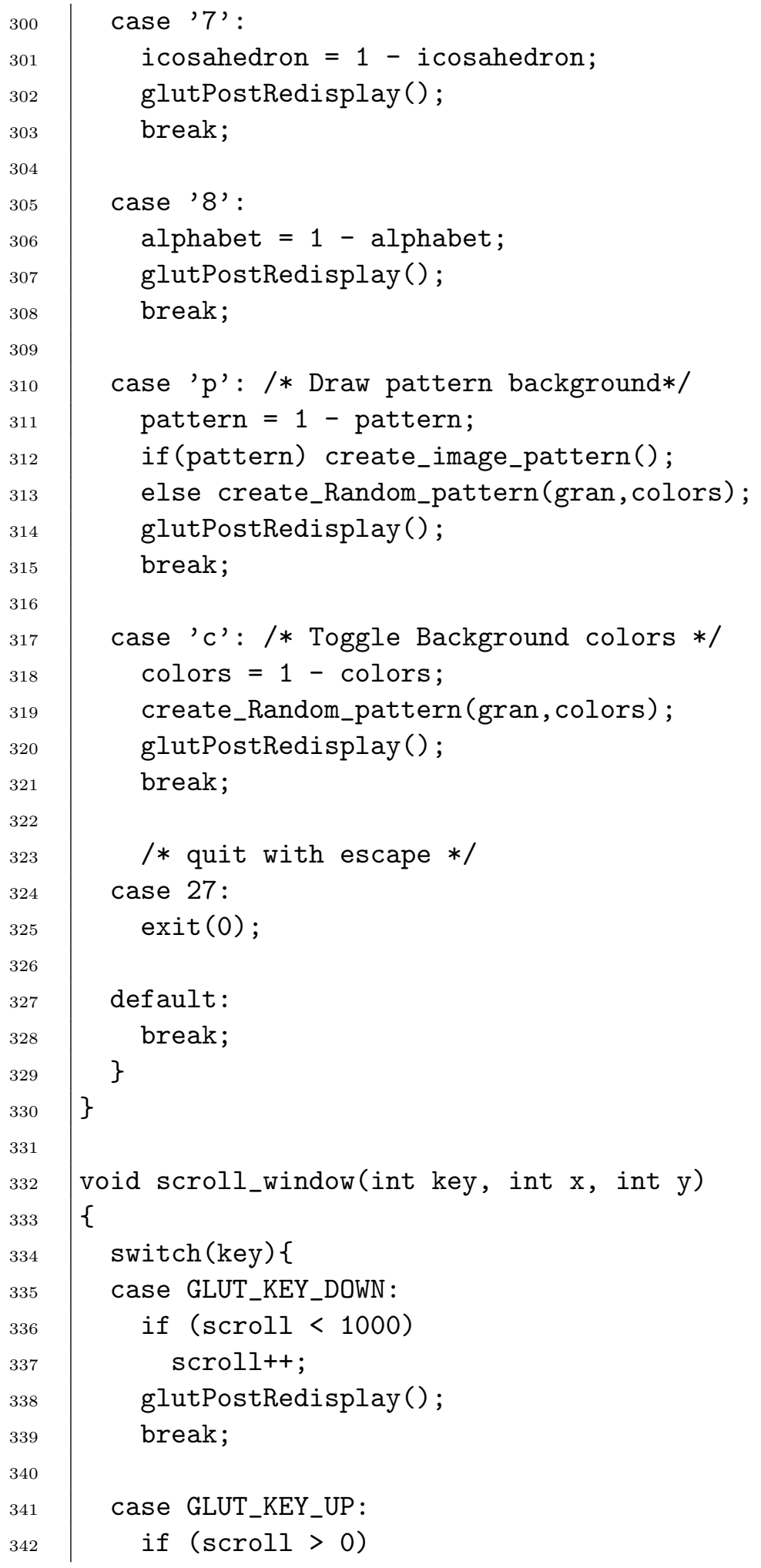




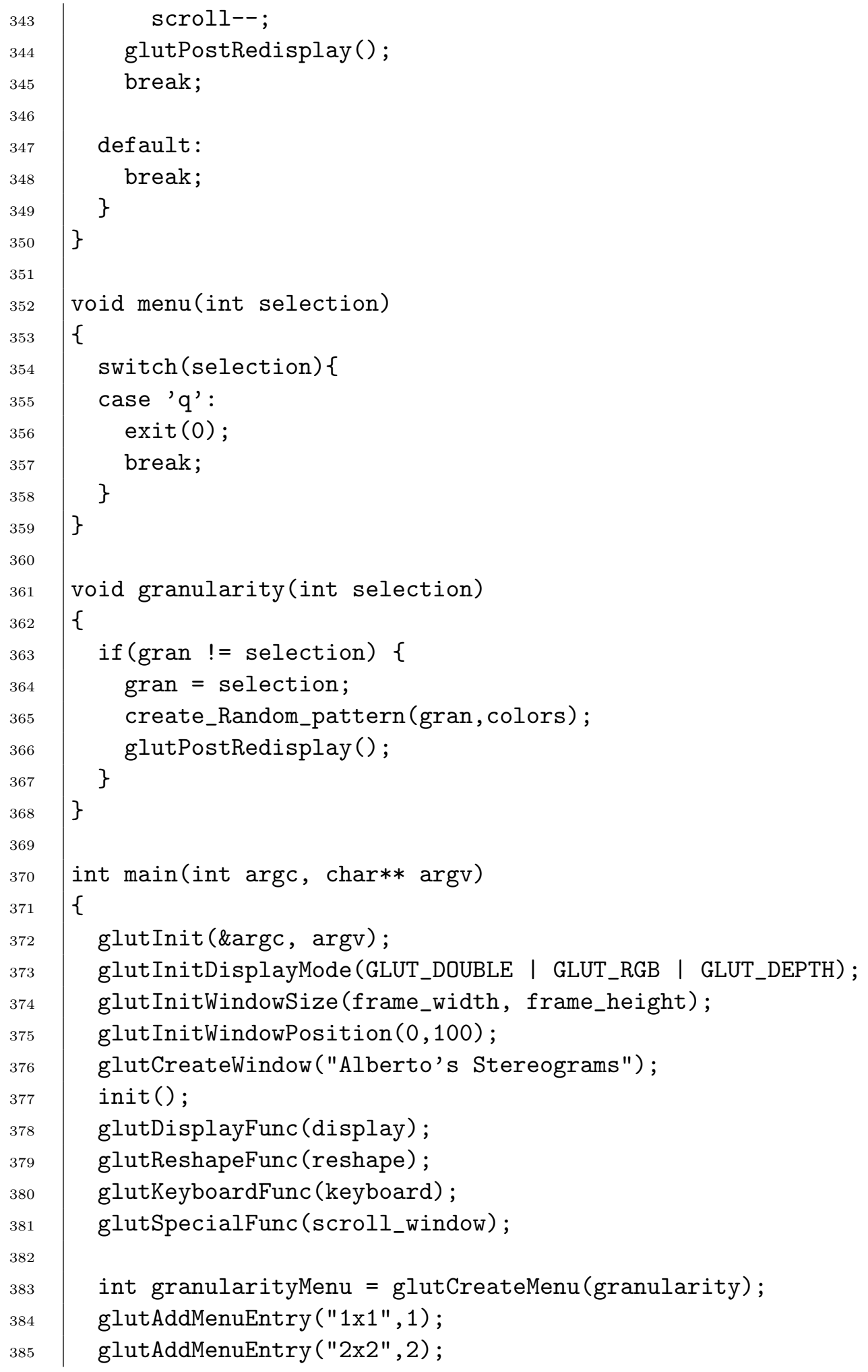




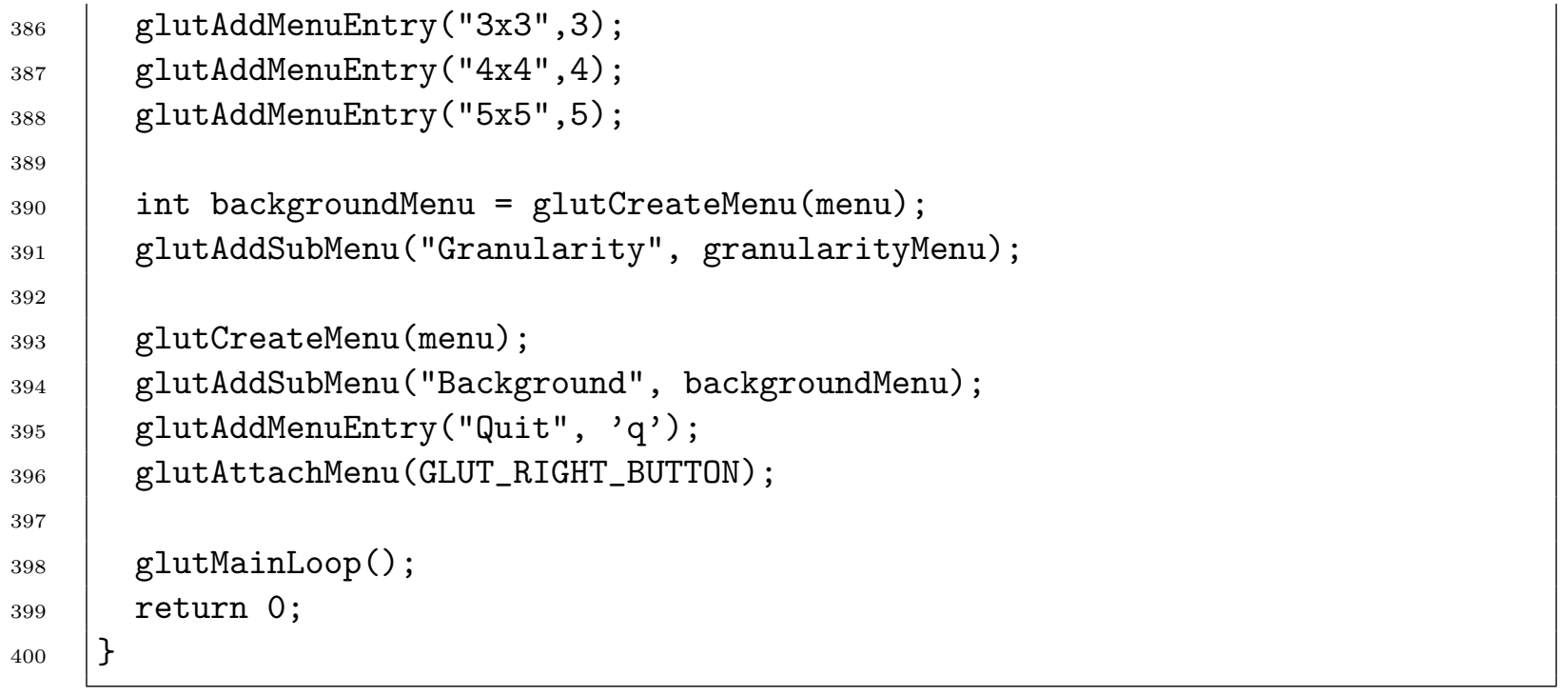




\section{Stereogram Story and Questionnaire}

\section{Questionnaire}

Please answer the following multiple choice questions based on your best recollection of the content of the material you have just read. Circle your answer or print in case of a blank.

1) The flames of fire are
A) Pink
B) Gray
C) Red \& Yellow
D) Other

2) Toi was born in
A) 1829
B) 1842
C) 1856
D) None of the above

3) The name of the character is Ro _ Doe
A) $\mathrm{F}$
B) A
C) $\mathrm{E}$
D) None of the above

4) Doe lived :
A) in a colorful hut next to a lake close to where a river meets the sea
B) in a colorful hut next to a lake
C) in a colorful hut at the valley of Porton

5)What Was Ro's Favorite seasoning
A) Salt
B) Saffron
C) Chocolate
D) None of the above

6)The chicken cooked for
A) 3 hours
B) 20 minutes
C) 30 minutes

7) What is the name of the lake?

8) What is the name of the Dog?

9) Rate the level of comfort while reading the SIRDS
A) total discomfort
B) partial discomfort
C) partially comfortable
D) highly comfortable 


\section{Story}

The following story will be presented in the autostereogram.

This is the story of a person named Ro N. Doe. She was born in the valley of Porton in the year 1857. Ro had a brother named Toi, who was born a year before. Doe lived in a colorful hut next to lake Chark. Chark was far away from where the river meets the sea.

Ro was a great cook. One evening Ro used a pan to roast a chicken for dinner. The chicken had Ro's favorite seasoning. After Ro sprinkled the poultry for 30 minutes, the chicken cooked for three hours.

Doggy was Ro's pet that usually ate leftovers of Ro's delicious cuisine. Doggy was no ordinary dog. He could eat dark chocolate. Usually Ro bought extra just for him.

That night, Toi launched julienned chocolate to space. The metallic blue flames of fire burned as the rocket headed towards the sun over a gray horizon.

Doggy, Toi and Ro enjoyed the chicken and the spectacle in the sky. 


\section{Experiment Data}

\begin{tabular}{|c|c|c|c|c|c|c|c|c|c|}
\hline \multicolumn{10}{|c|}{ Questionnaire Data } \\
\hline Participant & \multicolumn{9}{|c|}{ Question Number } \\
\hline Female $q$ Male $\sigma^{7}$ & 1 & 2 & 3 & 4 & 5 & 6 & 7 & 8 & 9 \\
\hline \multicolumn{10}{|c|}{ Monocular Group } \\
\hline q & B & $\mathrm{C}$ & $\mathrm{D}$ & A & $\mathrm{D}$ & A & $\emptyset$ & Toi & $\mathrm{C}$ \\
\hline 9 & $\mathrm{~B}$ & $\mathrm{C}$ & $\mathrm{D}$ & $\mathrm{A}$ & $\emptyset$ & A & Crake & Doggy & $\mathrm{B}$ \\
\hline q & $\mathrm{A}$ & $\mathrm{C}$ & $\mathrm{D}$ & B & $\mathrm{D}$ & A & Charie & Doggy & $\mathrm{C}$ \\
\hline q & D (No text) & $\mathrm{C}$ & $\mathrm{D}$ & $\mathrm{A}$ & $\mathrm{D}$ & A & Chark & Doggie & $\mathrm{B}$ \\
\hline q & $\mathrm{C}$ & $\mathrm{D}$ & $\mathrm{D}$ & $\mathrm{A}$ & $\mathrm{C}$ & A & Chark & Doggy & $\mathrm{C}$ \\
\hline q & blue & $\mathrm{D}$ & $\mathrm{D}$ & $\mathrm{A}$ & $\mathrm{D}$ & A & Porton & Doggie & $\mathrm{D}$ \\
\hline q & Blue & $\mathrm{D}$ & $\mathrm{C}$ & B & $\mathrm{D}$ & A & Tig & Doggy & $\mathrm{D}$ \\
\hline$\sigma^{\prime \prime}$ & B & B & $\mathrm{D}$ & $\mathrm{A}$ & $\mathrm{A}$ & A & Saffron & Doggy & $\mathrm{C}$ \\
\hline$\sigma^{\prime}$ & D (No text) & $\mathrm{A}$ & $\mathrm{D}$ & $\mathrm{A}$ & $\mathrm{D}$ & $\mathrm{C}$ & chu chu & Doggy & $\mathrm{B}$ \\
\hline$\sigma^{\prime}$ & B & $\mathrm{C}$ & $\mathrm{D}$ & $\mathrm{C}$ & $\mathrm{C}$ & A & Chark & Doggy & $\mathrm{C}$ \\
\hline$\sigma^{\prime \prime}$ & Blue & $\mathrm{D}$ & $\mathrm{D}$ & B & $\mathrm{D}$ & A & Chark & $\emptyset$ & $\mathrm{C}$ \\
\hline$\sigma^{\prime \prime}$ & $\mathrm{C}$ & $\mathrm{D}$ & $\emptyset$ & $\mathrm{A}$ & $\mathrm{C}$ & A & $\emptyset$ & $\emptyset$ & $\mathrm{C}$ \\
\hline$\sigma^{\prime}$ & blue & $\mathrm{C}$ & $\mathrm{C}$ & B & $\mathrm{C}$ & A & Charcon & Doggie & $\mathrm{D}$ \\
\hline$\sigma^{\prime \prime}$ & blue & $\mathrm{D}$ & $\mathrm{D}$ & $\mathrm{C}$ & $\mathrm{C}$ & $\mathrm{A}$ & Chark & Doggy & $\mathrm{C}$ \\
\hline$\sigma^{\prime \prime}$ & $\mathrm{C}$ & $\mathrm{C}$ & $\mathrm{B}$ & $\mathrm{C}$ & A & A & Porton Lake & Toei & $\mathrm{B}$ \\
\hline \multicolumn{10}{|c|}{ Stereographic Group } \\
\hline q & $\mathrm{C}$ & $\mathrm{B}$ & $\mathrm{D}$ & $\mathrm{C}$ & $\mathrm{A}$ & $\mathrm{A}$ & $\emptyset$ & Bud & $\mathrm{C}$ \\
\hline q & $\emptyset$ & $\mathrm{D}$ & $\mathrm{C}$ & $\mathrm{C}$ & $\emptyset$ & $\mathrm{A}$ & $\emptyset$ & Doggy & $\mathrm{B}$ \\
\hline q & $\mathrm{A}$ & $\mathrm{B}$ & $\mathrm{C}$ & $\mathrm{A}$ & $\mathrm{B}$ & $\mathrm{A}$ & $\emptyset$ & Doggy & $\mathrm{B}$ \\
\hline q & blue & $\mathrm{B}$ & $\mathrm{C}$ & $\mathrm{A}$ & $\mathrm{C}$ & $\mathrm{A}$ & $\emptyset$ & Doggy & $\mathrm{B}$ \\
\hline q & $\mathrm{C}$ & $\mathrm{C}$ & B & $\mathrm{C}$ & $\mathrm{C}$ & $\mathrm{C}$ & Chart & Doggy & $\emptyset$ \\
\hline q & B & $\mathrm{D}$ & $\mathrm{D}$ & B & $\mathrm{D}$ & $\mathrm{A}$ & $\emptyset$ & Doggie & $\mathrm{B}$ \\
\hline q & blue & $\mathrm{A}$ & $\mathrm{D}$ & $\mathrm{B}$ & $\mathrm{D}$ & $\mathrm{A}$ & $\emptyset$ & Doggy & $\mathrm{C}$ \\
\hline Q* & $\mathrm{A}$ & A & B & $\mathrm{A}$ & $\mathrm{D}$ & B & $\emptyset$ & $\emptyset$ & $\mathrm{A}$ \\
\hline$\sigma^{\prime \prime}$ & blue & $\mathrm{D}$ & $\mathrm{D}$ & B & $\mathrm{C}$ & $\mathrm{A}$ & $\emptyset$ & Doggy & $\mathrm{C}$ \\
\hline$\sigma^{\prime \prime}$ & $\emptyset$ & $\mathrm{D}$ & $\mathrm{C}$ & B & $\mathrm{C}$ & $\mathrm{A}$ & $\emptyset$ & Doggy & $\mathrm{C}$ \\
\hline$\sigma^{\prime \prime}$ & blue & $\mathrm{C}$ & $\mathrm{D}$ & B & $\mathrm{D}$ & $\mathrm{A}$ & Chark & Doggy & $\mathrm{C}$ \\
\hline$\sigma^{\prime \prime}$ & B & $\mathrm{C}$ & $\mathrm{D}$ & $\mathrm{A}$ & $\mathrm{C}$ & $\mathrm{C}$ & $\emptyset$ & Doggy & $\mathrm{B}$ \\
\hline$\sigma^{\prime \prime}$ & metallic blue & $\mathrm{D}$ & $\mathrm{D}$ & B & $\emptyset$ & $\mathrm{A}$ & $\emptyset$ & Doggy & $\mathrm{B}$ \\
\hline$\sigma^{\prime \prime}$ & Blue & $\mathrm{C}$ & $\mathrm{D}$ & B & $\mathrm{C}$ & $\mathrm{A}$ & Chark & Doggy & $\mathrm{D}$ \\
\hline$\sigma^{\prime \prime}$ & $\mathrm{C}$ & $\mathrm{D}$ & $\mathrm{D}$ & $\mathrm{A}$ & B & $\mathrm{A}$ & $\emptyset$ & Tot & $\mathrm{B}$ \\
\hline$\sigma^{\prime \prime}$ & Blue & $\mathrm{C}$ & $\mathrm{D}$ & $\mathrm{C}$ & $\mathrm{D}$ & $\mathrm{A}$ & $\emptyset$ & Doggy & $\mathrm{B}$ \\
\hline
\end{tabular}

